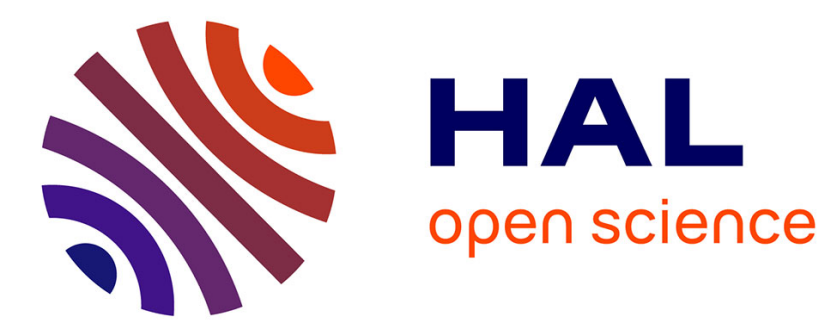

\title{
Discrete Topological Transformations for Image Processing
}

Michel Couprie, Gilles Bertrand

\section{To cite this version:}

Michel Couprie, Gilles Bertrand. Discrete Topological Transformations for Image Processing. Brimkov, Valentin E. and Barneva, Reneta P. Digital Geometry Algorithms, 2, Springer, pp.73-107, 2012, Lecture Notes in Computational Vision and Biomechanics, 978-94-007-4174-4. 10.1007/978-94007-4174-4_3. hal-00727377

\section{HAL Id: hal-00727377 https://hal.science/hal-00727377}

Submitted on 3 Sep 2012

HAL is a multi-disciplinary open access archive for the deposit and dissemination of scientific research documents, whether they are published or not. The documents may come from teaching and research institutions in France or abroad, or from public or private research centers.
L'archive ouverte pluridisciplinaire HAL, est destinée au dépôt et à la diffusion de documents scientifiques de niveau recherche, publiés ou non, émanant des établissements d'enseignement et de recherche français ou étrangers, des laboratoires publics ou privés. 


\title{
Chapter 3 \\ Discrete Topological Transformations for Image Processing
}

\author{
Michel Couprie and Gilles Bertrand
}

\begin{abstract}
Topology-based image processing operators usually aim at transforming an image while preserving its topological characteristics. This chapter reviews some approaches which lead to efficient and exact algorithms for topological transformations in 2D, 3D and grayscale images. Some transformations which modify topology in a controlled manner are also described. Finally, based on the framework of critical kernels, we show how to design a topologically sound parallel thinning algorithm guided by a priority function.
\end{abstract}

\subsection{Introduction}

Topology-preserving operators, such as homotopic thinning and skeletonization, are used in many applications of image analysis to transform an object while leaving unchanged its topological characteristics. In particular, skeletons are often used as a simplification of the original data, which facilitates shape recognition, registration or animation.

In this chapter, we will see how to define and efficiently implement such operators, on the basis of elementary topology-preserving transformations. We will also discuss some geometrical aspects of skeletons, as well as the need for filtering them. Besides, we will see that it is sometimes interesting to be able to selectively modify topology: we will present, in particular, a method that suppresses holes (or tunnels) in 3D images, depending on a "size" criterion.

Michel Couprie

Université Paris-Est, Laboratoire d'Informatique Gaspard-Monge, Équipe A3SI, ESIEE

Paris, France, e-mail: m.couprie@esiee.fr

Gilles Bertrand

Université Paris-Est, Laboratoire d'Informatique Gaspard-Monge, Équipe A3SI, ESIEE

Paris, France, e-mail: g.bertrand@esiee.fr 
These transformations are usually defined for acting on binary images (i.e., pixel or voxel sets). In Sect. 3.2, we will extend them to the case of grayscale images (i.e., functions), and present some applications to image filtering, segmentation and restoration.

There are two main kinds of thinning procedures: the sequential ones, that take a single point in consideration at each step, and the parallel ones, that attempt at removing a whole set of points at each iteration. In the first case, the result most often depends on the order in which the points are considered, while the latter kind permits to provide a well-defined result, which is generally more robust than the former to noise and orientation changes. The third part of this chapter deals with parallel thinning: we present the framework of critical kernels, that provides a mean to guarantee the topological soundness of parallel homotopic transformations. We introduce in this framework a new algorithm that builds at once a well-defined family of filtered Euclidean skeletons.

\subsection{Topological Transformations of Binary Images}

\subsubsection{Neighborhoods, Connectedness}

First of all, let us recall the basic definitions of digital topology [34, 29] that will be used in this chapter.

A point $x \in \mathbb{Z}^{D}(D=2,3)$ is defined by $\left(x_{1}, \ldots, x_{D}\right)$ with $x_{i} \in \mathbb{Z}$. We consider the neighborhood relations $N_{4}$ and $N_{8}$ defined for any point $x \in \mathbb{Z}^{2}$ by:

$N_{4}(x)=\left\{y \in \mathbb{Z}^{2} ;\left|y_{1}-x_{1}\right|+\left|y_{2}-x_{2}\right| \leqslant 1\right\}$, $N_{8}(x)=\left\{y \in \mathbb{Z}^{2} ; \max \left(\left|y_{1}-x_{1}\right|,\left|y_{2}-x_{2}\right|\right) \leqslant 1\right\}$,

and the neighborhood relations $N_{6}, N_{26}$ and $N_{18}$ defined for any point $x \in \mathbb{Z}^{3}$ by:

$N_{6}(x)=\left\{y \in \mathbb{Z}^{3} ;\left|y_{1}-x_{1}\right|+\left|y_{2}-x_{2}\right|+\left|y_{3}-x_{3}\right| \leqslant 1\right\}$,

$N_{26}(x)=\left\{y \in \mathbb{Z}^{3} ; \max \left(\left|y_{1}-x_{1}\right|,\left|y_{2}-x_{2}\right|,\left|y_{3}-x_{3}\right|\right) \leqslant 1\right\}$

$N_{18}(x)=\left\{y \in N_{26}(x) ;\left|y_{1}-x_{1}\right|+\left|y_{2}-x_{2}\right|+\left|y_{3}-x_{3}\right| \leqslant 2\right\}$.

These neighborhoods are illustrated in Fig. 3.1.

In the sequel, we denote by $n$ a number such that $n \in\{4,8,6,26\}$. We define $N_{n}^{*}(x)=N_{n}(x) \backslash\{x\}$. The point $y \in E$ is said to be $n$-adjacent to the point $x \in E$ if $y \in N_{n}^{*}(x)$. An $n$-path is an ordered sequence of points $x_{0} \ldots x_{k}$ such that $x_{i}$ is $n$-adjacent to $x_{i-1}$ for any $i \in\{1, \ldots, k\}$.

We denote by $E$ the set $\mathbb{Z}^{2}$ or $\mathbb{Z}^{3}$. Let $X \subseteq E$, we say that two points $x, y$ of $X$ are $n$-connected in $X$ if there exists an $n$-path in $X$ between those two points. This defines an equivalence relation on $X$. The equivalence classes for this relation are the $n$-connected components of $X$ (see Fig. 3.2). A subset $X$ 

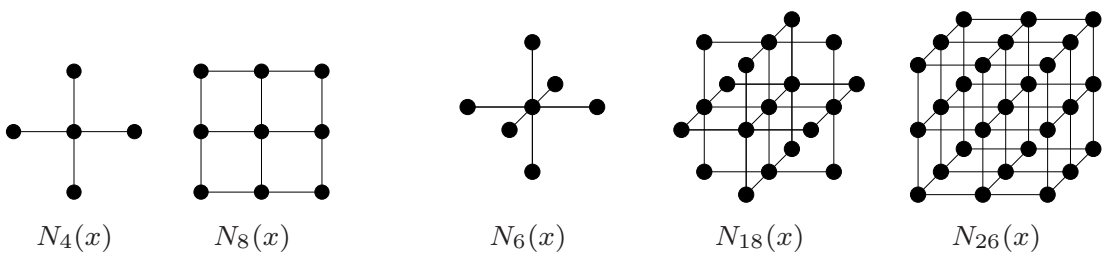

Fig. 3.1 Different neighborhoods of a point $x$ (the central point) in $2 \mathrm{D}$ and in $3 \mathrm{D}$.

of $E$ is said to be $n$-connected if it is composed of exactly one $n$-connected component.
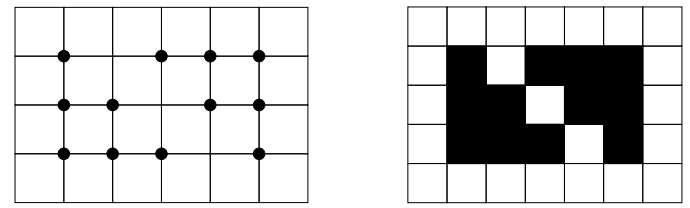

Fig. 3.2 The set of black points has two 4-connected components, and only one 8connected component. This figure also illustrates two common representations of a binary digital image (points on the left, pixels on the right).

The set composed of all $n$-connected components of $X$ is denoted by $C_{n}(X)$. A subset $Y$ of $E$ is said to be $n$-adjacent to a point $x \in E$ if there exists a point $y \in Y$ that is $n$-adjacent to $x$. The set of all $n$-connected components of $X$ that are $n$-adjacent to $x$ is denoted by $C_{n}^{x}(X)$. Remark that $C_{n}(X)$ and $C_{n}^{x}(X)$ are sets of subsets of $X$, and not sets of points. Besides, if $S$ is a finite set, we denote by $|S|$ the number of elements of $S$.

\subsubsection{Connectivity Numbers}

Intuitively, a point $x$ of an object $X \subseteq E$ is said to be simple if it can be deleted from $X$ while preserving the topological characteristics of $X$ (see [19]). In the case of $\mathbb{Z}^{2}$, this implies preserving the number of connected components of both the object and its complementary set. In $\mathbb{Z}^{3}$, it is necessary to preserve also holes (or tunnels), a notion that may be formalized through the fundamental group (see e.g. [24]).

Note that the definition of a simple point relies on notions (connected components, tunnels) that can be classified as global, in the sense that they cannot be defined without taking the whole object into account. Nevertheless, we will see that in $2 \mathrm{D}$ and $3 \mathrm{D}$, it is possible to characterize simple points 
on a local basis, thanks to the connectivity numbers introduced in this section. Such a local characterization is essential to get efficient algorithms for topological transformations.

Let $X$ be an open bounded subset of $\mathbb{R}^{D}$, and let $\lambda \in \mathbb{R}, \lambda \geqslant 0$. We denote by $\bar{X}$ the complement set of $X$, i.e., $\bar{X}=\mathbb{R}^{D} \backslash X$. If we use a $n$-connectivity for $X$ then we have to use a $\bar{n}$-connectivity for $\bar{X}$. For example in 2D the 4-connectivity for $X$ is associated with the 8-connectivity for $\bar{X}$, and in 3D the 6-connectivity for $X$ is associated with the 26-connectivity for $\bar{X}$. This is necessary to have a correspondence between topological characteristics of $X$ and $\bar{X}$ (see e.g. [29]). To summarize, we have the following possibilities in $2 \mathrm{D}:(n, \bar{n})=(4,8)$ or $(8,4)$; and in $3 \mathrm{D}^{1}:(n, \bar{n})=(6,26)$ or $(26,6)$.

Now, we can define the connectivity numbers in $2 \mathrm{D}$ and in $3 \mathrm{D}$ [3]. Intuitively, the connectivity number of a point $x$ relative to a set $X$, counts the number of connected components of $X \backslash\{x\}$, which are in the neighborhood of $x$, and which are adjacent to $x$.

Definition 1. Let $X \subseteq \mathbb{Z}^{2}$ and $x \in \mathbb{Z}^{2}$. Let $n \in\{4,8\}$. The (2D) connectivity numbers are defined as follows:

$$
\begin{aligned}
& T_{4}(x, X)=\left|C_{4}^{x}\left[N_{8}^{*}(x) \cap X\right]\right|, \\
& T_{8}(x, X)=\left|C_{8}^{x}\left[N_{8}^{*}(x) \cap X\right]\right| .
\end{aligned}
$$

In Fig. 3.3, we illustrate some connectivity numbers in 2D. Figure 3.3b shows the neighborhhod of point $u$, we can verify that $T_{8}(u, X)=1$ and $T_{4}(u, \bar{X})=1$. Similarly, the reader can check that $T_{8}(v, X)=T_{4}(v, \bar{X})=1$. For pixel $x$, we have $T_{8}(x, X)=T_{4}(x, \bar{X})=2$ (see Fig. 3.3c). The same holds for pixel $y$.

Definition 2. Let $X \subseteq \mathbb{Z}^{3}$ and $x \in \mathbb{Z}^{3}$. The (3D) connectivity numbers are defined as follows:

$$
\begin{aligned}
T_{6}(x, X) & =\left|C_{6}^{x}\left[N_{18}^{*}(x) \cap X\right]\right|, \\
T_{26}(x, X) & =\left|C_{26}^{x}\left[N_{26}^{*}(x) \cap X\right]\right| .
\end{aligned}
$$

Figure 3.4 shows some examples that illustrate this definition. Note that components that are not adjacent to the central point, according to the chosen adjacency relation, are not taken into account: this is illustrated in Fig. 3.4b.

\subsubsection{Topological Classification of Object Points}

If we use the $n$-connectivity for $X$ and the $\bar{n}$-connectivity for $\bar{X}$, the numbers $T_{n}(x, X)$ and $T_{\bar{n}}(x, \bar{X})$ give us topological characteristics of the point $x$ in the object $X$. In particular, the connectivity numbers allow us to detect whether a point is simple or not $[3,10]$, both in $2 \mathrm{D}$ and in $3 \mathrm{D}$ :

1 For the sake of simplicity we do not discuss here the case of the 18-connectivity, see $[32,3,10]$ for more information. 


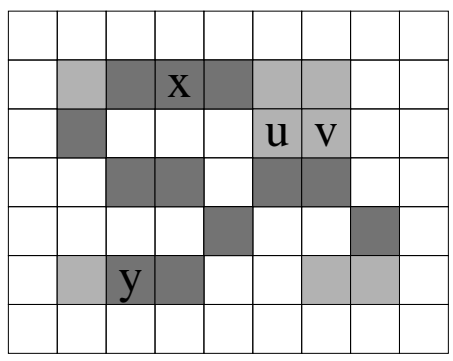

(a)

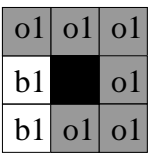

(b)

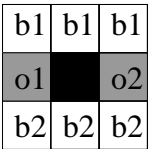

(c)

Fig. 3.3 We set $(n, \bar{n})=(8,4)$. (a): An object $X$ (light gray and dark gray pixels). (b): The eight neighbors of pixel $u$. The unique 8-connected component of $N_{8}^{*}(u) \cap X$ is labelled with $o 1$, and the unique 4-connected component of $N_{8}^{*}(u) \cap \bar{X}$ is labelled with $b 1$. (c): Depicts the eight neighbors of pixel $x$ or pixel $y$. The 8-connected components of $N_{8}^{*}(x) \cap X$ are labelled with $o 1, o 2$, and the 4-connected components of $N_{8}^{*}(x) \cap \bar{X}$ are labelled with $b 1, b 2$.

Theorem 1. Let $X \subseteq E$ and $x \in X$. The point $x$ is n-simple if and only if $T_{n}(x, X)=1$ and $T_{\bar{n}}(x, \bar{X})=1$.

Intuitively, this characterization states that a point is simple if and only if there is, in its neighborhood, exactly one "object" component and one "background" component. For example, in Fig. 3.3a, we conclude from the computation of connectivity numbers that points $u, v$ are both simple, whereas $x, y$ are both non-simple points. In this figure, all simple points are in lighter gray.

Note that the neighborhoods of points $x$ and $y$ are the same (Fig. 3.3c), hence also the connectivity numbers, but different events occur whenever $x$ or $y$ is deleted from $X$. In the case of $x$, two background components are merged; whereas if $y$ disappears, $X$ is splitted into two components. Note also that any simple point may be removed from $X$ without altering topology, but removing simultaneously $u$ and $v$ for instance would change topological characteristics of the image (here, the number of background components). We will see in Sect. 3.4.5 how to perform parallel thinning with topological guarantees.

The characterization of Theorem 1 also holds in the 3D case, see the examples of Fig. 3.4.

The fact that an intrinsically global notion - the one of simple pointadmits a local characterization is indeed a quite remarkable property. It will allow us to efficiently implement topological transformations.

The connectivity numbers are also useful to detect other kinds of points of particular interest. A point $x$ such that $T_{n}(x, X)=0$ is an isolated point. If $T_{\bar{n}}(x, \bar{X})=0$, then we have an interior point. The border points are characterized by $T_{\bar{n}}(x, \bar{X}) \neq 0$. 
(a)
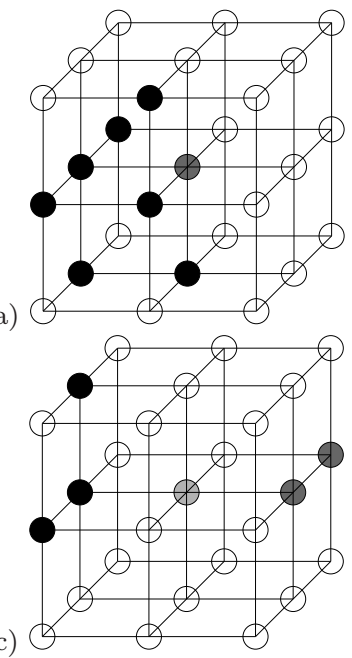

(b)

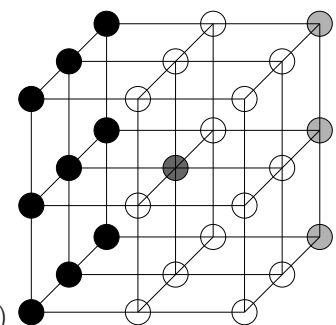

(d)

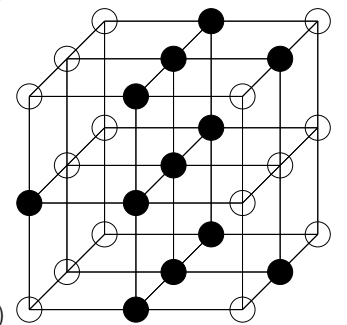

Fig. 3.4 (a): The central point $x$ is a 6 -simple point $\left(T_{6}(x, X)=T_{26}(x, \bar{X})=1\right)$ : the unique "object" component in its neighborhood is in black, and the unique "background" component is in white. We have also $T_{26}(x, X)=T_{6}(x, \bar{X})=1$, hence $x$ is 26-simple. (b): The central point $x$ is a 6 -simple point $\left(T_{6}(x, X)=T_{26}(x, \bar{X})=1\right)$ : there are two "object" components in its neighborhood, but only the one in black is 6-adjacent to $x$. However, $x$ is not 26 -simple, for $T_{26}(x, X)=2$. (c): The central point $x$ is such that $T_{6}(x, X)=2$ and $T_{26}(x, \bar{X})=1$; the two "object" components are in black and dark gray. (d): The central point $x$ is such that $T_{6}(x, X)=1$ and $T_{26}(x, \bar{X})=2$.

Let us consider the case where $E=\mathbb{Z}^{3}$, and take a point $x$ such that $T_{n}(x, X) \geqslant 2$. If we delete $x$ from $X$, we locally disconnect the object $X$ (see Fig. 3.4b). We say that such a point is a $1 D$ isthmus.

Consider the simplest case where $T_{n}(x, X)=2$ (see an example in Fig. 3.4c). Two situations may occur whenever $x$ is deleted. In the first case, the two local components involved in the definition of $T_{n}(x, X)$ are in fact connected together by a path in $X$ outside the neighborhood of $x$, and the deletion of the latter suppresses a tunnel from the object (this situation is similar to the one of point $x$ in Fig. 3.3a, in 2D). In the second case, the two local components are not connected and the deletion of $x$ indeed disconnects the object (see $y$ in Fig. 3.3a for a similar 2D situation). In both cases, topology is not preserved, in other words the point $x$ is not simple.

In the same way, a point $x$ such that $T_{\bar{n}}(x, \bar{X}) \geqslant 2$ is called a $2 D$ isthmus; its deletion causes the merging of connected components of the neighborhood of $x$ in $\bar{X}$ (see Fig. 3.4d). If these components are connected together in $\bar{X}$, the deletion of $x$ creates a new tunnel for the object, and if they are not, the deletion of $x$ causes decrease of the number of cavities. Also here, the point $x$ is non-simple. 


\subsubsection{Topology-preserving Transformations}

Deleting a simple point from an object $X$ yields an object $Y$ included in $X$, which is "topologically equivalent" to $X$. If we iterate this elementary operation, we can obtain a family of nested sets that are all topologically equivalent to $X$. More formally, we say that $Y$ is an elementary homotopic thinning of $X$, and we write $X \stackrel{e}{\rightarrow} Y$, if there exists a simple point $x$ for $X$ such that $Y=X \backslash\{x\}$. We say that $Y$ is a homotopic thinning of $X$ if $Y=X$ or if there exists a sequence $\left\langle Y_{0}, \ldots, Y_{k}\right\rangle$ such that $Y_{0}=X, Y_{k}=Y$ and $Y_{0} \stackrel{e}{\rightarrow} \ldots \stackrel{e}{\rightarrow} Y_{k}$. If, furthermore, no point in $Y$ is simple, we say that $Y$ is an ultimate homotopic thinning of $X$.

When transforming an object $X$ through an homotopic thinning, it is often needed to preserve from deletion a given subset $K$ of $X$. Such a subset is called a constraint set, and we say that $Y$ is an homotopic thinning of $X$ constrained by $K$ if $Y$ is an homotopic thinning of $X$ such that $K \subseteq Y$. If, furthermore, no point of $Y \backslash K$ is simple, we say that $Y$ is an ultimate homotopic thinning of $X$ constrained by $K$.

In order to thicken an object $X$ in a topology-preserving manner, it is sufficient to compute an homotopic thinning of the complementary set of $X$ (for the dual connectivity), and to take the complementary of the result.

\subsubsection{Transformations Guided by a Priority Function}

The order in which points are considered during a thinning process plays, of course, an important role with respect to the geometrical aspect of the result. This order can be specified by means of a numerical function, called priority function.

With each point $x$ of $X$, a priority function associates an integer or real number $P(x)$, which represents the priority of point $x$. The points of $X$ will be treated during the thinning process following the increasing values of $P$. To certain points $x$, a value $P(x)=+\infty$ may be given, meaning that these points must be preserved; in other words, the points with infinite priority constitute the constraint set.

This strategy is realized by the Algorithm GuidedThinning. The complexity of this algorithm is determined by the choice of the data structure used to represent the function $P$. For example, a balanced search tree allows for reaching a global time complexity in $O(n \log n)$, where $n$ is the number of image points. In certain particular cases, including the very common case where the function $P$ is a distance map [35], it is possible to implement Algorithm GuidedThinning in linear time (see [1]).

If one wants to use Algorithm GuidedThinning for skeletonization purposes, a natural choice for the priority function is a distance map relative to the background. In other words, the points with highest priority (i.e., small- 


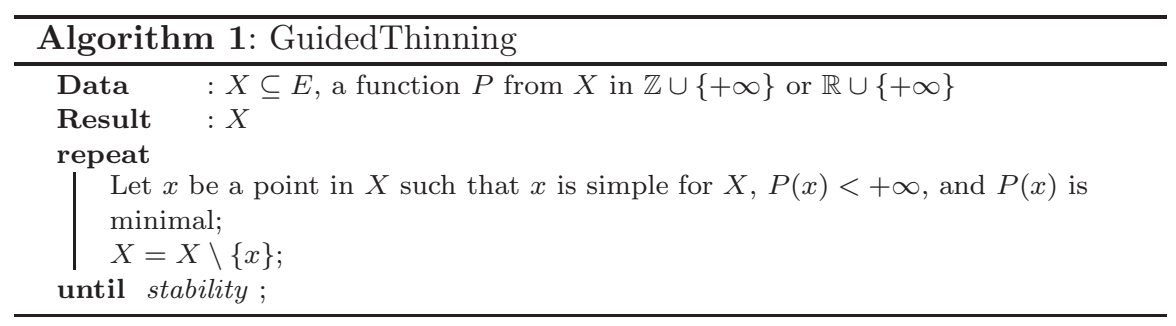

est value) are those closest to the background, and the points that "survive" are well centered in the object, in the sense that their distance to the background is, roughly speaking, maximal. Note that any distance may be chosen: discrete distances [35], chamfer distances [13], Euclidean distance [23], etc. The choice of the Euclidean distance permits to obtain the lowest sensibility to rotations.

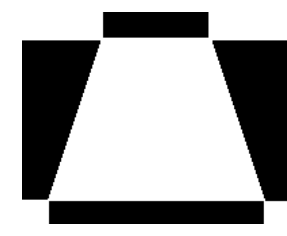

(a)

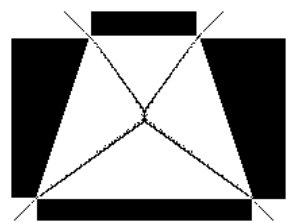

(b)

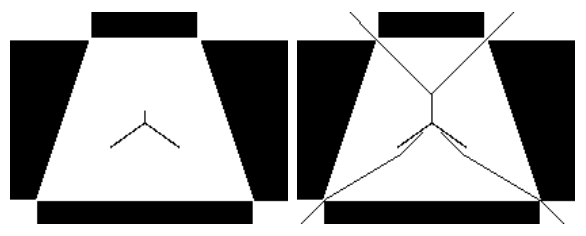

(c)

(d)

Fig. 3.5 (a): The original object $X$ (in white). (b): The Euclidean medial axis of $X$ (centers of maximal balls, see text), superimposed to $X$. (c): A subset $Y$ of the medial axis. (d): Result of the skeletonization using the Euclidean distance map as a priority function, and $Y$ as constraint set.

However, choosing the exact Euclidean distance map as a priority function for removing simple points from the object may lead to geometric distortions [38]. To illustrate this point, let us consider the object $X$ depicted in white in Fig. 3.5a. In Fig. 3.5b, we show in black superimposed to $X$, all the centers of maximal included Euclidean balls (that is, balls that are included in $X$ but which are not subsets of any other ball included in $X$ ). This is one of the possible definitions for the medial axis of $X$ (see also Sect. 3.2.6). It is usual to take only a subset of the medial axis as constraint set for computing centered skeletons, since the full medial axis often contains spurious points. Such a constraint set, let us call it $Y$, is depicted in Fig. 3.5c, superimposed to $X$. We use as priority function the map $P$ defined by

$P(x)= \begin{cases}+\infty & \text { whenever } x \in Y ; \\ d(x, \bar{X}) & \text { otherwise }\end{cases}$ 
where $d(x, \bar{X})=\min \{d(x, y) \mid y \in \bar{X}\}$, and $d(x, y)$ denotes the Euclidean distance between $x$ and $y$. When $Y=\emptyset$, the function $P$ is just the distance map relative to $X$.

Figure 3.5d depicts the result of Algorithm GuidedThinning in this case. Note that the obtained skeleton deviates from the medial axis points.

In the next section, we will study another priority function that gives better results than the Euclidean distance map, and is linked to a family of filtered medial axes.

\subsubsection{Lambda-medial Axis}

The notion of medial axis has been introduced by Blum in the 60s [11, 12]. In the continuous Euclidean space, the following definition can be used to formalise this notion: let $X$ be a bounded subset of $\mathbb{R}^{n}$, the medial axis of $X$ consists of the points $x \in X$ that have more than one nearest points on the boundary of $X$.

A major difficulty when using the medial axis in applications (e.g., shape recognition), is its sensitivity to small contour perturbations, in other words, its lack of stability. A recent survey [2] summarises selected relevant studies dealing with this topic. Because of this problem, it is usually necessary to add a filtering step (or pruning step) to any method that aims at computing the medial axis.

In 2005, F. Chazal and A. Lieutier introduced the $\lambda$-medial axis [16], a particular class of filtered skeletons, and studied its properties, in particular those related to stability. A major outcome of [16] is the following property: informally, except for particular values of the filtering parameter, the $\lambda$-medial axis remains stable under perturbations of the shape that are small with regard to the Hausdorff distance.

The original definition of the $\lambda$-medial axis (see [16]) holds and makes sense in the (continuous) Euclidean $D$-dimensional space.

Let $x=\left(x_{1}, \ldots, x_{D}\right), y=\left(y_{1}, \ldots, y_{D}\right) \in \mathbb{R}^{D}$, we denote by $d(x, y)$ the Euclidean distance between $x$ and $y$, in other words, $d(x, y)=\left(\sum_{k=1}^{D}\left(y_{k}-\right.\right.$ $\left.\left.x_{k}\right)^{2}\right)^{\frac{1}{2}}$. Let $S \subseteq \mathbb{R}^{D}$, we set $d(y, S)=\min _{x \in S}\{d(y, x)\}$.

Let $x \in \mathbb{R}^{D}, r \in \mathbb{R}, r \geqslant 0$, we denote by $B_{r}(x)$ the ball of radius $r$ centered on $x$, defined by $B_{r}(x)=\left\{y \in \mathbb{R}^{D} \mid d(x, y) \leqslant r\right\}$.

Let $S$ be a nonempty subset of $\mathbb{R}^{D}$, and let $x \in \mathbb{R}^{D}$. The projection of $x$ on $S$, denoted by $\Pi_{S}(x)$, is the set of points $y$ of $S$ which are at a minimal distance from $x$; more precisely,

$$
\Pi_{S}(x)=\{y \in S \mid \forall z \in S, d(y, x) \leqslant d(z, x)\} .
$$

The $\lambda$-medial axis of $X$ is the set of points $x$ in $X$ such that the radius of the smallest ball that includes $\Pi_{\bar{X}}(x)$ is not less than $\lambda$. For example in Fig. 3.6, we show a shape that is an ellipsis with a small "bump", and we 
consider the interior $X$ of this shape. Two different $\lambda$-medial axes of $X$ are displayed on the right.
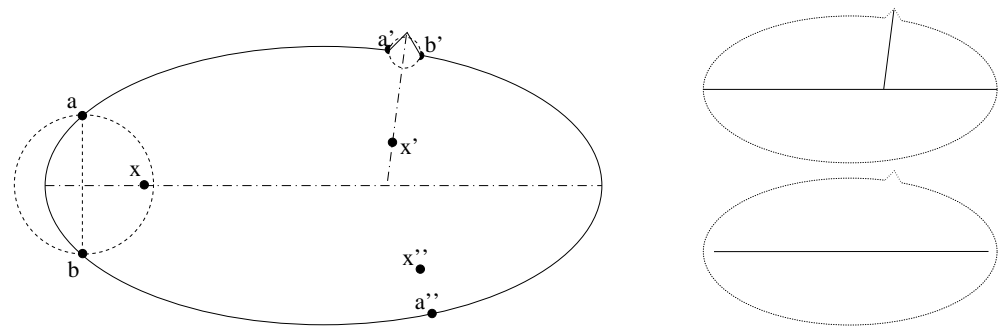

Fig. 3.6 Illustration of the $\lambda$-medial axis in $\mathbb{R}^{2}$. Left: Points $x, x^{\prime}$ and $x^{\prime \prime}$ and their respective closest boundary points. Top right: $\lambda$-medial axis with $\lambda=\epsilon$, a very small positive real number. Bottom right: $\lambda$-medial axis with $\lambda=d\left(a^{\prime}, b^{\prime}\right)+\epsilon$.

Now, let us consider the discrete case. For each point $x \in \mathbb{Z}^{D}$, we define the direct neighborhood of $x$ as $N(x)=\left\{y \in \mathbb{Z}^{D} \mid d(x, y) \leqslant 1\right\}$. Thus, $N(x)=N_{4}(x)\left(\right.$ resp. $\left.N_{6}(x)\right)$ whenever $D=2($ resp. $D=3)$.

Transposing directly the definition of the $\lambda$-medial axis to the discrete grid $\mathbb{Z}^{D}$ would yield unsatisfactory results. For instance, consider a horizontal ribbon in $\mathbb{Z}^{2}$ with constant, even width and infinite length (see Fig. 3.7). Clearly, the projection of any point of this set on its complementary set is reduced to a singleton. If we keep the same definition as above, any $\lambda$-medial axis of this object with $\lambda>0$ would be empty.

\begin{tabular}{|l|l|l|l|l|l|l|l|l|}
\hline & & & A & C & B & & & \\
\hline & & & & c & & & & \\
\hline & & & a & X & b & & & \\
\hline & & & & d & & & & \\
\hline & & & & & & & & \\
\hline & & & & D & & & & \\
\hline
\end{tabular}

Fig. 3.7 We consider a object $X$ in $\mathbb{Z}^{2}$ that is a horizontal ribbon of infinite length and width 4 (partially depicted here in gray). The projection of $x$ on $\bar{X}$ is $\Pi_{\bar{X}}(x)=\{C\}$. The smallest ball that includes $\Pi_{\bar{X}}(x)$ is the one with center $C$ and radius 0 . The projections of $a, b, c, d$ on $\bar{X}$ are respectively $\{A\},\{B\},\{C\},\{D\}$. Hence, the extended projection of $x$ on $\bar{X}$ is $\Pi \frac{e}{\bar{X}}(x)=\{A, B, C, D\}$, and we have $P R_{X}(x)=R>2$. The pixels in darker gray are in any $\lambda$-medial axis with $\lambda \leqslant R$, those in lighter gray are only in the 0 -medial axis of $X$.

This is why we need the following notion. Let $X \subseteq \mathbb{Z}^{D}$, and let $x \in X$. The extended projection of $x$ on $\bar{X}$ (where $\bar{X}=\mathbb{Z}^{D} \backslash \bar{X}$ ), denoted by $\Pi_{\bar{X}}(x)$, 
is the union of the sets $\Pi_{\bar{X}}(y)$, for all $y$ in $N(x)$ such that $d(y, \bar{X}) \leqslant d(x, \bar{X})$. Figure 3.7 illustrates this notion and the following ones.

Let $X$ be a finite subset of $\mathbb{Z}^{D}$, and let $\lambda \in \mathbb{R}, \lambda \geqslant 0$. We define the function $P R_{X}$ which associates, to each point $x$ of $X$, the value $P R_{X}(x)$ that is the radius of the smallest ball enclosing all the points of the extended projection of $x$ on $\bar{X}$. In other terms, $P R_{X}(x)=\min \left\{r \in \mathbb{R}, r \geqslant 0 \mid \exists y \in \mathbb{R}^{D}, \Pi \frac{e}{X}(x) \subseteq\right.$ $\left.B_{r}(y)\right\}$, and we call $P R_{X}(x)$ the projection radius of $x$ (for $\left.X\right)$.

The following definition was introduced in [14], together with an experimental evaluation of the stability and rotation invariance of the discrete $\lambda$-medial axis.

Definition 3 ([14]). The discrete $\lambda$-medial axis of $X$, denoted by $D L M A(X, \lambda)$, is the set of points $x$ in $X$ such that $P R_{X}(x) \geqslant \lambda$.

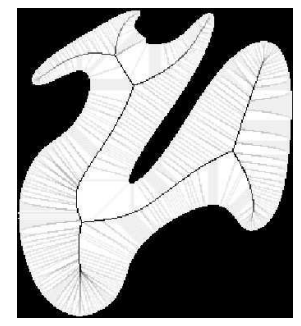

(a)

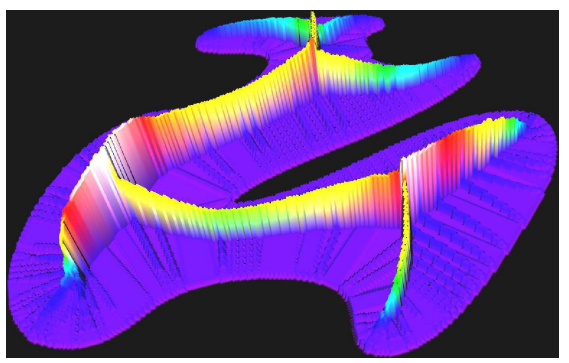

(b)

Fig. 3.8 (a): The function $P R_{X}$ superimposed to the shape $X$. Darkest gray levels represent highest values of $P R_{X}(x)$. (b): A $3 \mathrm{D}$ representation of the function $P R_{X}$.

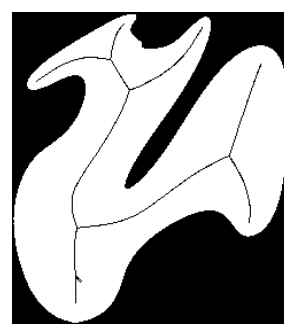

(a)

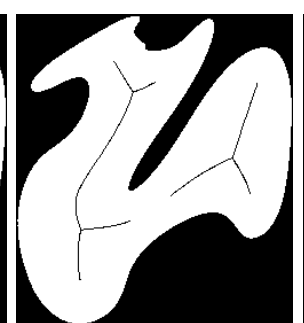

(b)

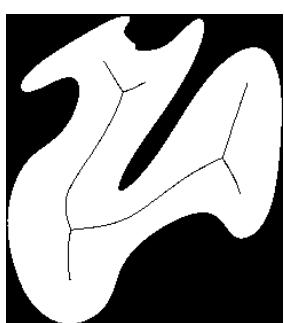

(c)

Fig. 3.9 Any DLMA of $X$ is a threshold of $P R_{X}$ at a particular value $\lambda$. (a): Discrete 7-medial axis. (b): discrete 25-medial axis of $X$. (c): Guided homotopic thinning of $X$, with $P R_{X}$ as priority function and with (b) as constraint set. 
Note that the function $P R_{X}$ can be computed once and stored as a grayscale image, and that any DLMA of $X$ is a level set of this function at a particular value $\lambda$ (see Fig. 3.8 and Fig. 3.9). For more details, illustrations and performance analysis, see [14].

The illustration on Fig. 3.9b is sufficient to demonstrate that a DLMA of a given shape $X$ may have a homotopy type different from the one of $X$.

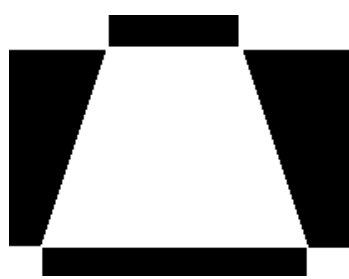

(a)

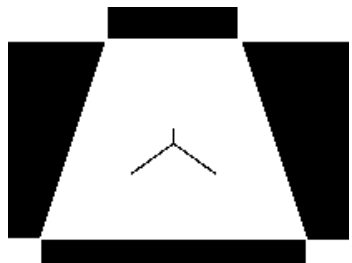

(b)

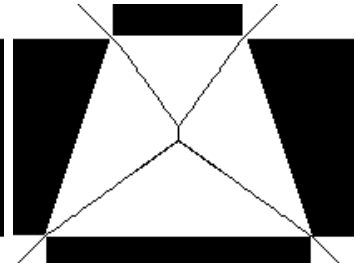

(c)

Fig. 3.10 (a): The original object $X$ (in white, the same as Fig. 3.5a). (b): A constraint set $Y$ : a filtered DLMA, that is also a set of centers of maximal included balls (see Fig. 3.5c). (c): Result of the skeletonization using $P R_{X}$ as a priority function, and $Y$ as constraint set.

The Algorithm GuidedThinning, with $P R_{X}$ as priority function and with a DLMA of $X$ as constraint set, provides filtered skeletons that are homotopic to $X$ and share the good geometric properties of the DLMAs (see Fig. 3.9c).

Another example is shown on Fig. 3.10, where a filtered Euclidean medial axis is used as a constraint set during skeletonization. As we have seen at the end of Sect. 3.2.5 (see also [38]), choosing the exact Euclidean distance map as a priority function for removing simple points from the object may lead to geometric distortions. In some cases, "extra branches" may even appear (see Fig. 3.5d). Choosing the map $P R_{X}$ as priority function yields more satisfying results (see Fig. 3.10c), as it guides the thinning process towards elements that belong to the different nested discrete $\lambda$-medial axes.

\subsubsection{Other Applications of Guided Thinning}

For certain applications, it may be relevant to take as priority function the gray levels of an image. This makes sense when these gray levels can be interpreted as a measure of the likelihood, for a pixel, to belong to a certain class or region.

To illustrate this, suppose that we want to extract from a $3 \mathrm{D}$ magnetic resonance image (MRI) of the head, the white matter of the brain (see Fig. 3.11a). From the knowledge of human anatomy and the parameters of the imagery device, we know that a volume element $x$ situated in the white 
matter produces a response that is coded by a value $F(x)$ for the corresponding voxel, which lies between two limits $\mu_{1}<\mu_{2}$. Assuming a Gaussian model, the voxels with value $\frac{\mu_{1}+\mu_{2}}{2}$ are those with highest probability to belong to the white matter. Furthermore, we know from anatomical data that the white matter of the brain constitutes a simply connected volume, in other words, it is topologically equivalent to a ball. In order to guarantee a result having the wanted topological characteristics, we use the following scheme: start with an object $X=\left\{x_{0}\right\}$, where $x_{0}$ is any point situated within the white matter; then perform an homotopic thinning of $\bar{X}$ (i.e. an homotopic thickening of $X)$ guided with the priority function $P$ defined by:

$P(x)= \begin{cases}|F(x)-\mu|, & \text { where } \mu=\frac{\mu_{1}+\mu_{2}}{2} \text { if } \mu_{1} \leqslant F(x) \leqslant \mu_{2}, \\ +\infty & \text { otherwise. }\end{cases}$

The values $+\infty$ ensure that all the resulting points have, in the image $F$, values that lie in the correct range (see Fig. 3.11b). This method has been successfully exploited to segment the white matter, as well as the cortex, from 3D MRI with topological guarantees [21, 22].

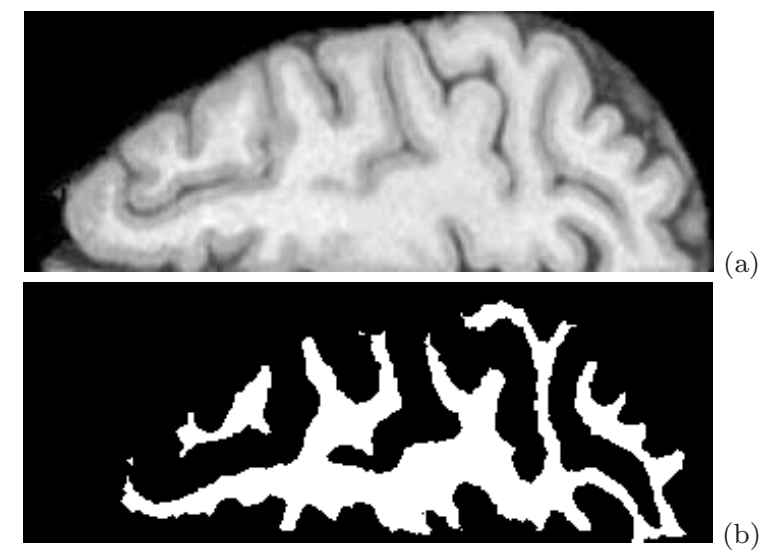

Fig. 3.11 (a): Detail of a 2D plane from a 3D MRI of the brain. (b): Result of the method described in the text. Note that the result is connected in $3 \mathrm{D}$, althought the shown $2 \mathrm{D}$ cross-section is not connected.

In this kind of application, it is useful to be able to apply morphological filtering operators (openings, closings, alternate sequential filters) on an object while guaranteeing topology preservation. See [18] for the definition of such filtering operators. 


\subsubsection{Hole Closing}

We have seen that it is possible, thanks to the notion of simple point, to design operators that transform an object while preserving its topological characteristics. However, controled topology modifications are needed in some applications. This topic is seldom addressed in the literature. In this section, we present a method [1] that is, to our knowledge, the first one that permits to close holes in a $3 \mathrm{D}$ object.

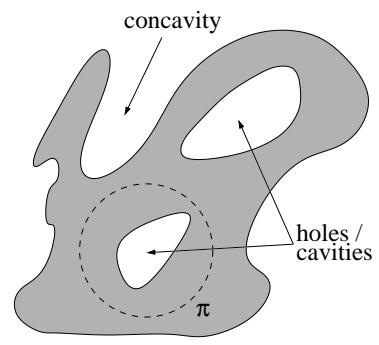

(a)

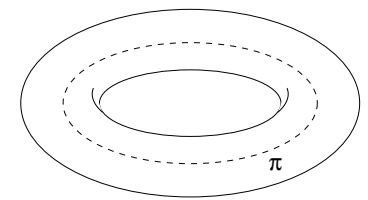

(b)

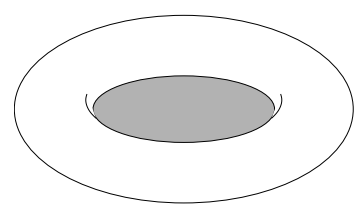

(c)

Fig. 3.12 (a): A 2D objects with two holes. (b): A solid torus. This object has one hole (tunnel), which is detected by the existence of path $\pi$. (c): The hole of the torus has been closed.

In our approach, we consider the notion of hole from a topological point of view. From this viewpoint, it is important to distinguish between holes, cavities and concavities. A concavity is a concave part of the contour of an object, it is not a topological feature. A cavity is a bounded connected component of the background, that forms a "hollow" in the object (see Fig. 3.12a).

A hole is much more complicated to define. Intuitively, the presence of a hole (or tunnel in 3D) in an object can be characterized by the existence of a closed path in the object that cannot be continuously deformed, inside the object, into a single point. For example in $3 \mathrm{D}$, a solid torus like the one depicted in Fig. 3.12b has one hole.

In $2 \mathrm{D}$, the notions of hole and cavity coincide, thus closing holes in $2 \mathrm{D}$ may be simply done by using algorithms for connected component extraction. But closing holes in 3D objects is by no means a trivial problem, because 3D holes are not, like in 2D, delimited regions of space.

Based on connectivity numbers (Sect. 3.2.2) and the strategy of guided thinning (Sect. 3.2.5), the method that we present here closes holes in any 3D object (see Fig. 3.13). In addition, this method allows for controlling the "size" of the holes that are to be closed (Fig. $3.13 b_{2,3}$ ). It can be implemented by a linear-time algorithm.

The basic idea of this method consists of embedding the object $X$, in which we want to close holes, into another object $Y$ that is connected, without any 
hole and without any cavity, such as a solid cuboid for example. Then, we iteratively shrink $Y$ by deleting points that do not belong to $X$, and ensuring thanks to the analysis of connectivity numbers that each point deletion does not create any hole or cavity. This method has been introduced and formalized in [1], we recall here its main notions and properties.

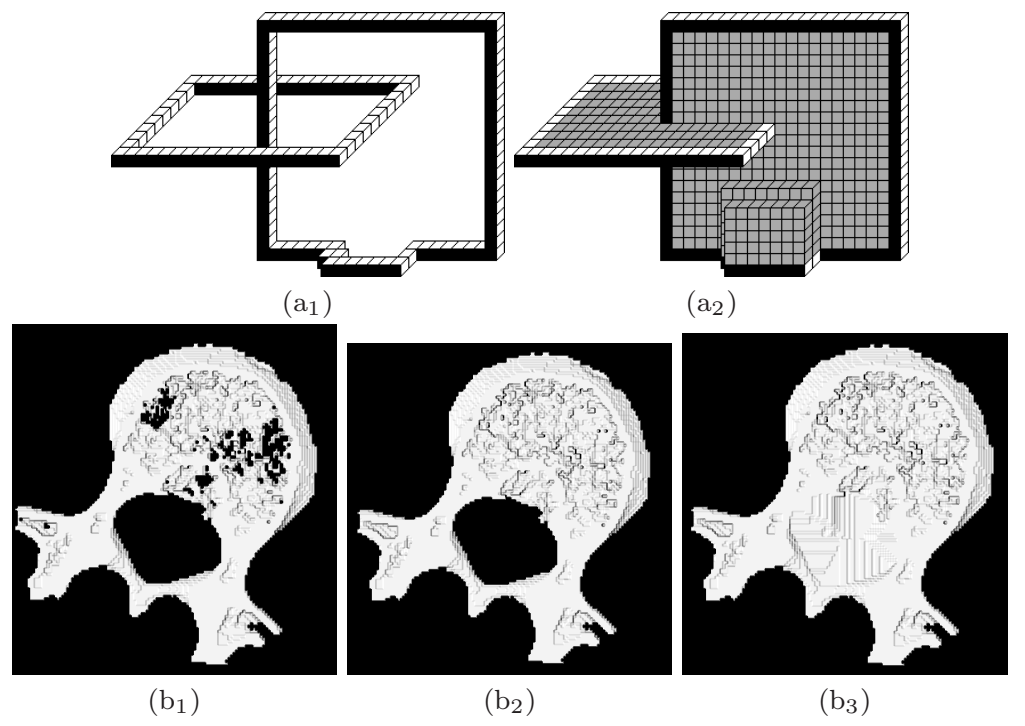

Fig. 3.13 Illustration of a hole closing algorithm for $3 \mathrm{D}$ objects. $\left(\mathrm{a}_{1}, \mathrm{a}_{2}\right)$ : The use of a distance map leads to a good centrering of the surface patch that closes the hole. $\left(b_{1}, b_{2}\right.$, $\left.\mathrm{b}_{3}\right)$ : A parameter controls the "size" of the holes to be closed.

Definition 4 ([1]). Let $X, Y$ be such that $X \subseteq Y \subseteq \mathbb{Z}^{3}$. We say that $Y$ is a topological hull of $X$ if $Y$ has no hole and no cavity, and if, for all $x \in Y \backslash X$, the set $Y \backslash\{x\}$ has a hole or a cavity.

For example in Fig. 3.13, $Y=\left(\mathrm{a}_{2}\right)$ is a topological hull of $X=\left(\mathrm{a}_{1}\right)$. The set $Y \backslash X$ (depicted by gray voxels in $\mathrm{a}_{2}$ ) corresponds to "surface patches" that close the holes.

The following theorem allows for a local characterization of the class of sets that are topological hulls, relatively to the class of sets that have no cavity and no hole.

Theorem 2 ([1]). Let $X, Y$ be such that $X \subseteq Y \subseteq \mathbb{Z}^{3}$. Suppose that $Y$ has no cavity and no hole. Then, $Y$ is a topological hull of $X$ if and only if, for each point $x$ of $Y \backslash X, x$ is an interior point or a $2 D$ isthmus for $Y$.

Corollary 1. Let $X, Y, Z$ be such that $X \subseteq Y \subseteq Z \subseteq \mathbb{Z}^{3}$, and such that $Z$ has no cavity and no hole. If $Y$ can be obtained from $Z$ by iterating the 
following two steps until stability:

- choose a point $x$ in $Z \backslash X$ such that $T_{\bar{n}}(x, \bar{Z})=1$;

- set $Z=Z \backslash\{x\}$

then $Y$ is a topological hull of $X$.

In order to get a result which is well-centered with respect to the object $X$, we use a distance map to guide this process, in the manner of Algorithm GuidedThinning. More precisely, the points in the complement of $X$ that are farthest from $X$ are treated in the first place. We can also use a parameter $s$ that allows for controlling the "size" of holes to be closed: if one also deletes, during the process, the candidate points $x$ that are such that $T_{\bar{n}}(x, \bar{X})>1$, and having a distance map value greater than $s$, then the biggest holes (in this sense) will be let open. The Algorithm HoleClosing formalizes this method. As for Algorithm GuidedThinning, with an adapted choice of data structure this algorithm may be implemented to run in linear time. Note that, whenever the parameter $s$ is set to $+\infty$, Algorithm HoleClosing indeed computes a topological hull of $X$ (in other words, it closes all holes).

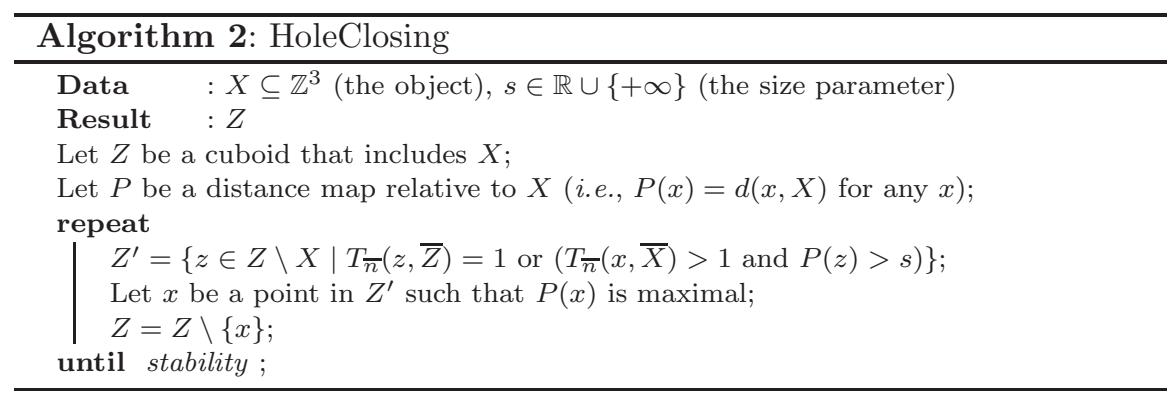

\subsection{Topological Transformations for Grayscale Images}

In this section topological notions such as those of simple point, homotopic thinning, ultimate homotopic thinning, are extended to the case of grayscale images. Applications to image filtering, segmentation and restoration are presented.

A 2D grayscale image can be seen as a function $F$ from $\mathbb{Z}^{2}$ into $\mathbb{Z}$. For each point $x$ of $\mathbb{Z}^{2}, F(x)$ is the gray level, or the luminosity of $x$. We denote by $\mathcal{F}$ the set of all functions from $\mathbb{Z}^{2}$ into $\mathbb{Z}$.

Let $F \in \mathcal{F}$ and $k \in \mathbb{Z}$, the cross-section (or threshold) of $F$ at level $k$ is the set $F_{k}$ composed of all points $x \in \mathbb{Z}^{2}$ such that $F(x) \geqslant k$. Observe that a cross-section is a set of points, i.e., a binary image. As for the binary case, if we use the $n$-adjacency for the cross-sections $F_{k}$ of $F$, we must use 
the $\bar{n}$-adjacency for the complementary sets $\overline{F_{k}}$, with $(n, \bar{n})=(8,4)$ ou $(4,8)$. Consider the function $-F$, that we call the complementary function of $F$ (for each point $x$ of $\left.\mathbb{Z}^{2},(-F)(x)=-F(x)\right)$. Note that the complementary sets of the cross-sections of $F$ are cross-sections of $-F$. In forthcoming examples and figures, we choose $n=8$ for the cross-sections of $F$, thus we must use $\bar{n}=4$ for the cross-sections of $-F$. A non-empty connected component $X$ of a cross-section $F_{k}$ of $F$ is a (regional) maximum for $F$ if $X \cap F_{k+1}=\emptyset$. A set $X \subseteq \mathbb{Z}^{2}$ is a (regional) minimum for $F$ if it is a regional maximum for $-F$.

\subsubsection{Cross-section Topology}

Intuitively, we say that a transformation of $F$ preserves topology if the topology of all cross-sections of $F$ is preserved. Hence, the "cross-section topology" of a function (i.e., of a grayscale image) directly derives from the topology of binary images [9]. Based on this idea, the following notions generalize the notion of simple point to the case of functions.

Definition 5. Let $F \in \mathcal{F}$, the point $x \in \mathbb{Z}^{2}$ is destructible (for $F$ ) if $x$ is simple for $F_{k}$, with $k=F(x)$. The point $x \in \mathbb{Z}^{2}$ is constructible (for $F$ ) if $x$ is destructible for $-F$.

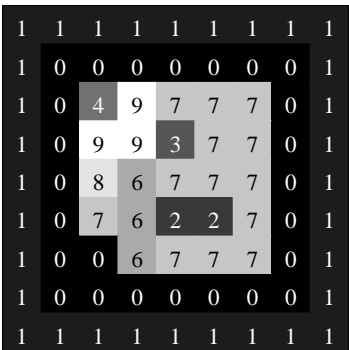

(a)

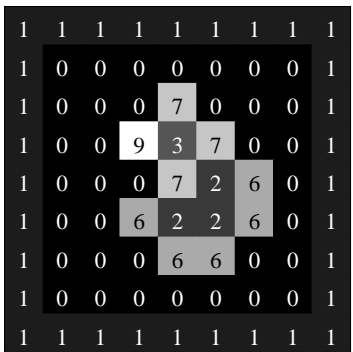

(b)

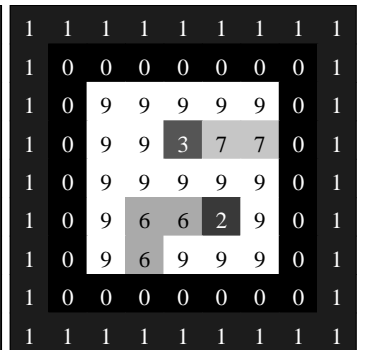

(c)

Fig. 3.14 (a): Original image. (b): An ultimate homotopic thinning of (a). (c): An ultimate homotopic thickening of (a).

We see that the gray level of a destructible (resp. constructible) point may be lowered (resp. raised) of one unit, while preserving the topology of $F$. For example in Fig. 3.14a, the point at level 8 is both destructible and constructible; the two points at level 2 are constructible, but only one of them may be raised, because after that, the other point would become non-constructible.

Let $F \in \mathcal{F}$ and $G \in \mathcal{F}$. We say that $G$ is an elementary homotopic thinning of $F$, and we write $F \stackrel{e}{\rightarrow} G$, if there exists a point $x$ that is destructible for 
$F$ such that $G(x)=F(x)-1$, and for each $y \neq x, G(y)=F(x)$. We say that $G$ is an homotopic thinning of $F$ if $G=F$ or if there exists a sequence $\left\langle G_{0}, \ldots, G_{k}\right\rangle$ such that $G_{0}=F, G_{k}=G$ and $G_{0} \stackrel{e}{\rightarrow} \ldots \stackrel{e}{\rightarrow} G_{k}$. Furthermore, if no point of $G$ is destructible, we say that $G$ is an ultimate homotopic thinning of $F$. We define in a dual manner the notions of homotopic thickening and ultimate homotopic thickening.

For example in Fig. 3.14, image (b) is an ultimate homotopic thinning of (a), and (c) is an ultimate homotopic thickening of (a).

\subsubsection{Local Characterizations and Topological Classification of Points}

Let $F \in \mathcal{F}$ and $x \in \mathbb{Z}^{2}$. For the sake of simplicity, we will omit to mention $F$ unless necessary; for example, we will write $N^{++}(x)$ rather than $N^{++}(x, F)$. We define the four neighborhoods:

$N^{++}(x)=\left\{y \in N_{8}^{*}(x) ; F(y)>F(x)\right\}$;

$N^{+}(x)=\left\{y \in N_{8}^{*}(x) ; F(y) \geqslant F(x)\right\}$;

$N^{--}(x)=\left\{y \in N_{8}^{*}(x) ; F(y)<F(x)\right\}$;

$N^{-}(x)=\left\{y \in N_{8}^{*}(x) ; F(y) \leqslant F(x)\right\}$.

We define also:

$\eta^{-}(x)= \begin{cases}\max \left\{F(y) ; y \in N^{--}(x)\right\} & \text { if } N^{--}(x) \neq \emptyset, \\ F(x) & \text { otherwise. }\end{cases}$

It is easy to show that lowering a destructible point $x$ down to the value $\eta^{-}(x)$ is a homotopic transformation. For example in Fig. 3.14a, the point at level 9 in the third row can be lowered down to 7 , then to 4 , and finally to 0 without changing the topology of cross-sections. This property, in addition to the local characterization of destructible and constructible points that we present next, allows for the design of efficient algorithms for computing transformations that preserve cross-section topology, on the model of e.g. Algorithm GuidedThinning (see [20]).

We define the four connectivity numbers:

$T^{++}(x)=\left|C_{n}\left[x, N^{++}(x)\right]\right| ; T^{+}(x)=\left|C_{n}\left[x, N^{+}(x)\right]\right| ;$

$T^{--}(x)=\left|C_{\bar{n}}\left[x, N^{--}(x)\right]\right| ; T^{-}(x)=\left|C_{\bar{n}}\left[x, N^{-}(x)\right]\right|$.

The following property can be straightforwardly derived from the above definition and from the local characterization of simple points in binary images (see Theorem 1). It shows that connectivity numbers allow for a local characterization of destructible and constructible points.

Let $F \in \mathcal{F}$ and $x \in \mathbb{Z}^{2}$.

$$
\begin{aligned}
& x \text { is destructible for } F \Leftrightarrow T^{+}(x)=1 \text { and } T^{--}(x)=1 ; \\
& x \text { is constructible for } F \Leftrightarrow T^{-}(x)=1 \text { and } T^{++}(x)=1 .
\end{aligned}
$$

Furthermore, connectivity numbers allow for a classification of topological characteristics of a point:

$x$ is a peak if $T^{+}(x)=0 ; x$ is minimal if $T^{--}(x)=0$; 
$x$ is $k$-divergent if $T^{--}(x)=k$ with $k>1$;

$x$ is a well if $T^{-}(x)=0 ; x$ is maximal if $T^{++}(x)=0$;

$x$ is $k$-convergent if $T^{++}(x)=k$ with $k>1$;

$x$ is a lower point if it is not maximal; $x$ is an upper point if it is not minimal;

$x$ is an interior point if it is both minimal and maximal;

$x$ is a simple side if it is both destructible and constructible;

$x$ is a saddle point if it is both convergent and divergent.

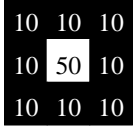

(a)

$\begin{array}{lll}10 & 10 & 10 \\ 10 & 10 & 10 \\ 10 & 10 & 10\end{array}$

(b)

\begin{tabular}{|lll|}
\hline 50 & 50 & 10 \\
50 & 50 & 10 \\
10 & 10 & 10 \\
\hline
\end{tabular}

(c)

$\begin{array}{|lll|}10 & 10 & 10 \\ 50 & 50 & 50 \\ 10 & 10 & 10\end{array}$

(d)

\begin{tabular}{|lll|}
\hline 50 & 30 & 50 \\
50 & 30 & 50 \\
10 & 10 & 10
\end{tabular}

(e) \begin{tabular}{|lrr|rrr|}
\hline 50 & 50 & 50 & 10 & 10 & 10 \\
50 & 30 & 10 & 50 & 30 & 50 \\
\hline 10 & 10 & 10 & 10 & 10 & 10 \\
\hline
\end{tabular}

(f) (g)

Fig. 3.15 Topological type. The central point has the following type: a: peak; b: interior; c: destructible maximal; d: maximal 2-divergent; e: destructible 2-convergent; f: simple side; g: saddle.

By considering all the possible values of the four connectivity numbers, one proves [9] that the type of a point $x \in \mathbb{Z}^{2}$, whatever the function $F \in \mathcal{F}$, is necesseraly one and only one of the following: 1) a peak; 2) a well; 3) an interior point; 4) a constructible minimal point; 5) a destructible maximal point; 6) a minimal convergent point; 7) a maximal divergent point; 8) a simple side; 9) a destructible convergent point; 10) a constructible divergent point; 11) a saddle point. Figure 3.15 shows examples of seven out of these eleven types; the four other types can be obtained by duality (for example a well is the dual of a peak, etc).

The rest of this chapter is devoted to three applications of cross-section topology. In these applications, we combine homotopic transformations and transformations that modify topology in a controlled manner.

\subsubsection{Topological Filtering}

In the case of impulse noise, a positive impulse takes the form of a small group of pixels, having grayscale values higher than those of pixels in their neighborhood. We can detect a positive impulse made of an isolated pixel $x$ by testing the topological type of $x$ : it is a peak. One can "destroy" this peak by lowering $x$ down to the value $\eta^{-}(x)$. For impulses formed by several adjacent pixels, this procedure is not sufficient. However, if we apply homotopic thinning to the image, an impulse formed by a few pixels may be reduced to a peak, allowing for its detection and deletion.

On the other hand, we do not want to lower bigger groups of pixels that may constitute significant objects in the image. This is why we need a notion 
of "thinning step" in order to control the spatial extent of the thinning (see [20] for more details).

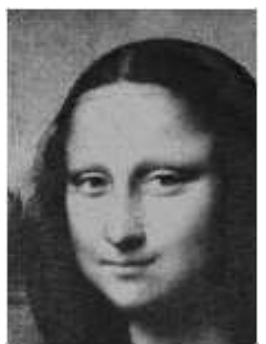

(a)

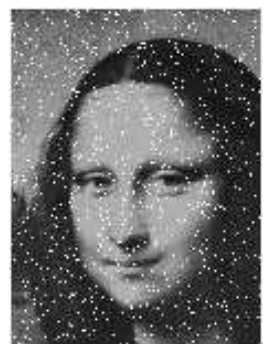

(b)

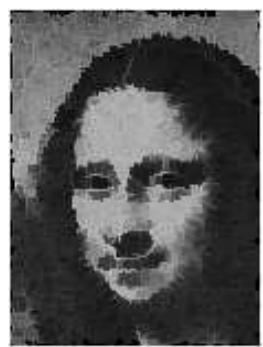

(c)

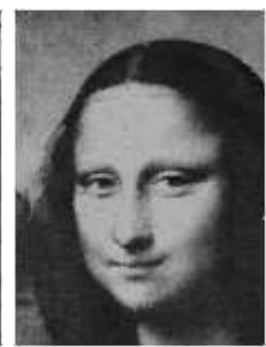

(d)

Fig. 3.16 Topological filtering. (a): Original image. (b): Original image with added impulse noise. (c): After 3 steps of homotopic thinning and peak lowering. (d): Homotopic reconstruction of (c) constrained by (b).

In Fig. 3.16, we show in (c) the result of three steps of homotopic thinning applied to image (b), followed by the lowering of all peaks. The positive impulses have been eliminated, but some points outside these impulses have also been lowered. It is thus necessary to restore the initial values of these points. We use for this purpose a homotopic reconstruction operator, which is nothing else but a homotopic thickening constrained by the original image (that is, the final value of a point cannot be higher than the value of this point in the original image). Since only constructible points can be raised, the lowered peaks will not be restored at their original value. Figure $3.16 \mathrm{~d}$ shows a homotopic reconstruction of (c) constrained by (b). Negative impulses can be filtered by the dual procedure. This topological filtering gives, for impulse noise, better results than a median filter or a filter based on morphological opening and reconstruction. In particular, it better preserves thin structures.

\subsubsection{Topological Segmentation}

Figure 3.17a shows an image in which one perceives dark cells separated by lighter borders. Due to noise, this image contains a lot of regional minima: they appear in white in (a'). An ultimate homotopic thinning (b) preserves, by construction, all these minima and extend them as much as possible (b'). Figure 3.18a shows a 1D profile extracted from such an ultimate homotopic thinning. In this profile, the points $A, B$ and $C$ correspond to divergent points that separate neighboring minima. Some of these divergent points $(A, B)$ can be considered as "irregular points" [9]: we would like to lower them in order to eliminate, by merging, some minima having small depth. 


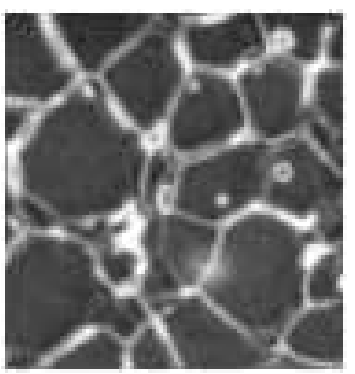

(a)

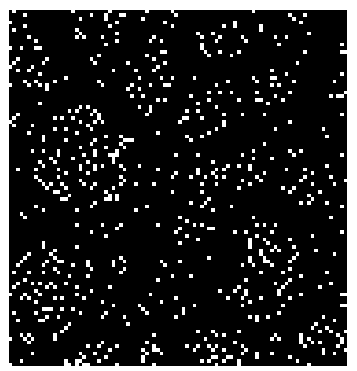

(a')

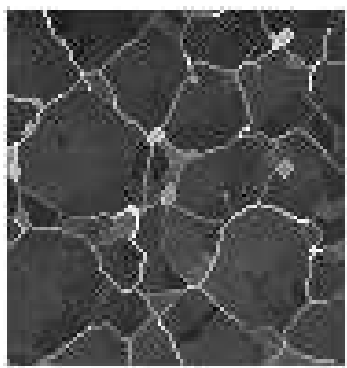

(b)

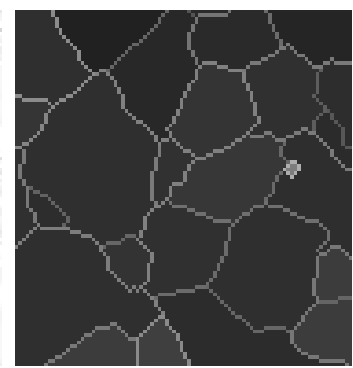

(c)

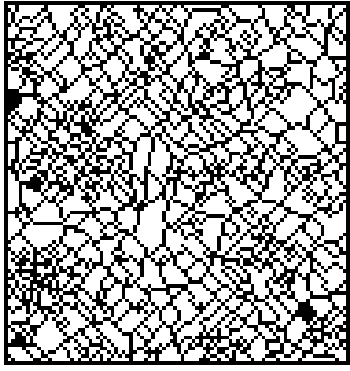

(b')

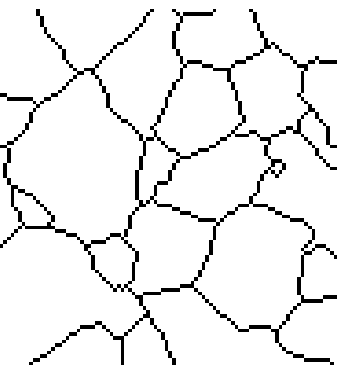

(c')

Fig. 3.17 Topological segmentation. (a): Original image. (b): Ultimate homotopic thinning. (c): Ultimate filtered thinning with $\kappa=40$. (a'), (b'), (c'): In white, the minima of (a), (b), (c) respectively.

To this aim, we introduce the notions of $\kappa$-destructible point and ultimate filtered thinning. Intuitively, a point $\kappa$-destructible $x$ is either a destructible point, or a peak, or a divergent point that lies on a crest line that divides its neighborhood into several lower regions, such that at most one of these regions has a difference of altitude with respect to $x$ that is greater than $\kappa$. Thus, the parameter $\kappa$ corresponds to a notion of local contrast. For example, points at levels 20 and 100 in Fig. 3.18b are both 10-destructible, but are not destructible. An ultimate filtered thinning is defined in a similar manner as an ultimate homotopic thinning, by using " $\kappa$-destructible" instead of "destructible".

In Fig. 3.17c, we see an ultimate filtered thinnning of (a) with $\kappa=40$. A binary segmented image (c') is obtained by extracting regional minima of (c). Note that this segmentation method involves only one parameter $(\kappa)$ relative to a notion of local contrast. 

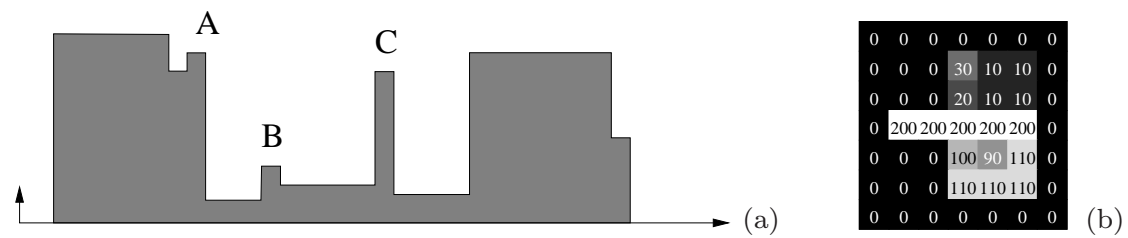

Fig. 3.18 Illustration of $\kappa$-destructible points. (a): A 1D profile of an ultimate homotopic thinning. (b): An image with two 10-destructible points (levels 20 and 100) that are not destructible.

\subsubsection{Crest Restoration Based on Topology}

Segmentation methods that are based on minima extraction and region merging, as well as those based on contour detection, are sensitive to the quality of the crests that separate the regions of interest (see Fig. 3.17, Fig. 3.20), which may be alterated by noise. In this section, we propose a procedure for detecting and eliminating narrow passes on the crests of a $2 \mathrm{D}$ function.

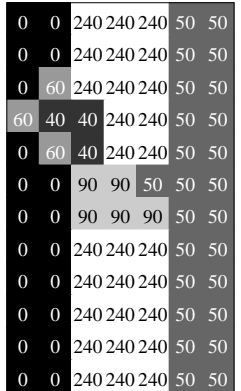

(a)

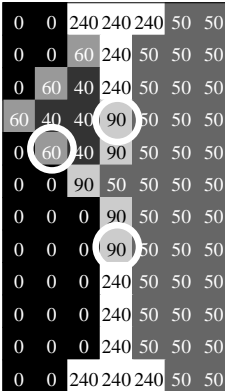

(b)

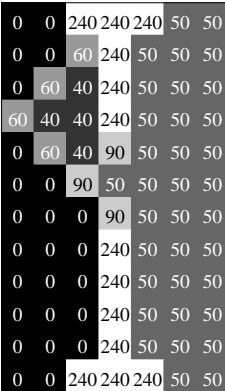

(c)

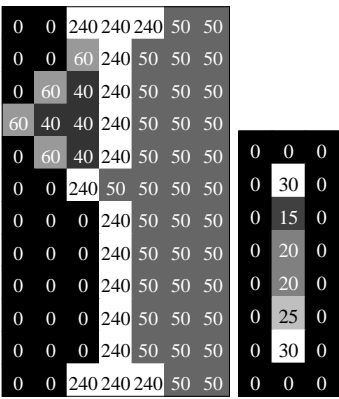

(d)

Fig. 3.19 Crest restoration. (a): The lowest value on the crest is the one of the pass (90). (b): After one step of homotopic thinning. (c,d): After 1 and 3 iterations of the crest restoration algorithm. (e): Points at levels 15, 20 and 25 are separating points.

First of all, we apply some steps of filtered or homotopic thinning, in order to reduce crests to thin lines (see Fig. 3.19b). After this, we can detect points that belong to "thin crests", and that must be raised in order to eliminate passes.

Let $X \subseteq \mathbb{Z}^{2}$ and $x \in X, x$ is a separating point (for $X$ ) if $\bar{T}(x) \geqslant 2$. Let $F \in \mathcal{F}$, a point $x \in \mathbb{Z}^{2}$ is called a separating point (for $F$ ) if there exists a level $k \in \mathbb{Z}$ such that $x$ is a separating point for the set $F_{k}$. Note that, if $x$ is a divergent point for $F$, then $x$ is necessarily a separating point for $F$, but the converse is not true. For example, in Fig. 3.19e, the points at levels 15, 
20 and 25 are separating points, whereas only the point at level 15 and the second point at level 20 (from the top) are divergent points.

We see in Fig. 3.19b that, in order to eliminate the pass at level 90, we can raise separating points that are constructible, until a saddle point appears. This saddle point can then be detected and raised. We also see in Fig. 3.19b that, if we iteratively raise constructible separating points without any restriction, we will also reinforce some low crest lines, like the one at level 60 . Indeed, the point at level 60 circled in white is a constructible separating point. Furthermore, we cannot use the notions of $\kappa$-destructible point and filtered thinnning in this case, because we would take the risk of lowering those very passes that we want to raise.

Now, let us define a class of points that are "good candidates" for crest restoration. Intuitively, such a point may be characterized by the presence, in its neighborhood, of a point $y$ which is a separating point for the section at level $k=F(x)$ but is not separating for higher sections. This is formalized through the notion of extensible point defined below.

Let $F \in \mathcal{F}$, a point $x \in \mathbb{Z}^{2}$ that is a separating point for $F$ is called extensible if it is, either a constructible point, of a saddle point for $F$, and if $x$ has at least one neighbor $y$ that satisfies the following two conditions:

i) $y$ is a separating point (in the binary sense) for $F_{k}$, with $k=F(x)$, and ii) $y$ is not a separating point (in the binary sense) for any cross-section $F_{j}$ with $j>k$.

For example in Fig. 3.19b, we can check that the two circled constructible points at level 90 are extensible, because each of them has a neighbour at 240 which is separating for $F_{90}$ but not for $F_{91}$ and higher sections; whereas the circled constructible point at level 60 is not extensible. Indeed, the point at 90 adjacent to the latter point is separating both for $F_{60}$ and for $F_{61}$.

The crest restoration method proceeds by iteratively detecting and raising extensible points. A more detailed description of the method can be found in [20]. In Fig. 3.19c, we see the result after applying one step of the method on (b). In particular, we see that two points at level 90 in (b) have been raised up to 240, and that points at level 60 have not been modified. In (d), we see the result after three iterations: the crest at 240 has been restored. Further iterations would not modify this result.

In Fig. 3.20, we illustrate this method on a gradient image (b). Image (b) is first thinned, giving (c). If we threshold this image, we see that either significant contour segments are lost (d), or we get too many details. Image (e) has been obtained from (c) by crest restoration until stability. The same threshold was applied on $(\mathrm{c}, \mathrm{e})$, giving $(\mathrm{d}, \mathrm{f})$ respectively. We see that many significant contour segments have been recovered, without introducing artefacts. 


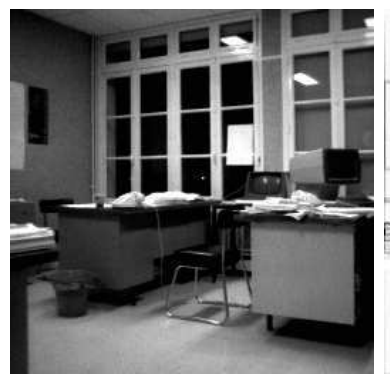

(a)

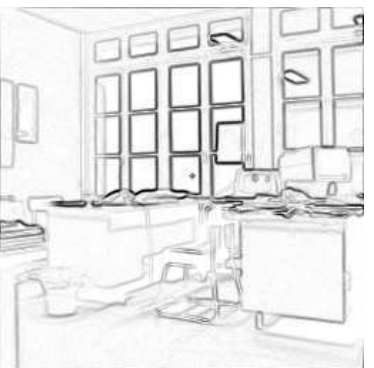

(b)

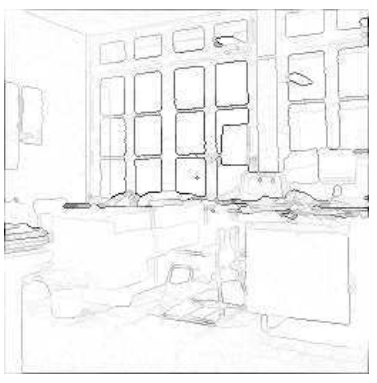

(c)

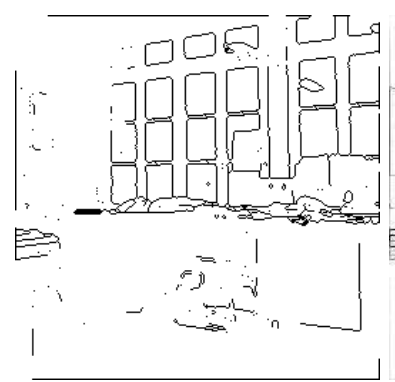

(d)

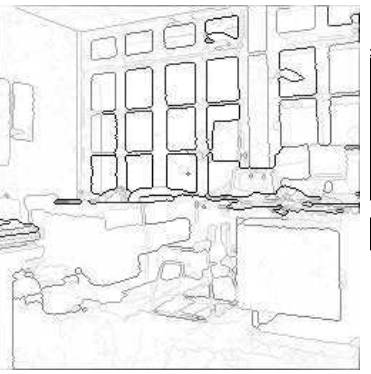

(e)

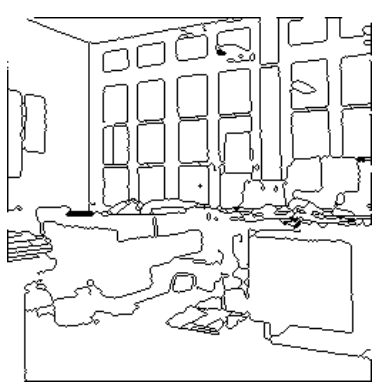

(f)

Fig. 3.20 Crest restoration. (a): Original image. (b): After applying a gradient modulus operator (the lowest values are in white). (c): After a filtered thinning. (e): After crest restoration, performed until stability. $(\mathrm{d}, \mathrm{f})$ : Thresholds of $(\mathrm{c}, \mathrm{e})$ respectively, at the same level.

\subsection{Parallel Thinning}

In Sects. 3.2 and 3.3, we described transformations that are sequential by nature. By this, we mean that after each point modification, the result of this modification has to be taken into account in order to perform simplicity tests for other points. Consequently, depending on the order in which the points are examined, some arbitrary choices may be done, and different results may be obtained depending on these choices. Even when one uses a priority function to guide the thinning, it is not seldom that many points share the same priority value, and arbitrary decisions are still necessary.

Another strategy for thinning objects consists of removing some of its border points in parallel $[36,37]$. However, parallel deletion of simple points does not, in general, guarantee topology preservation: see for example Fig. 3.3a, where removing both simple points $u, v$ would merge two components of the background. In fact, such a guarantee is not obvious to obtain, even for the 2D case (see [17], where fifteen published parallel thinning algorithms are analyzed, and counter-examples are shown for five of them). 
In order to study the conditions under which points may be removed simultaneously while preserving topology of $2 \mathrm{D}$ objects, C. Ronse introduced minimal non-simple sets [33]. This work leads to verification methods for the topological soundness of parallel thinning algorithms. Such methods have been proposed for 2D algorithms by C. Ronse [33] and R. Hall [25], they have been developed for the 3D case by T.Y. Kong [26, 27] and C.M. Ma [31]. For the $3 \mathrm{D}$ case, one of the authors [4] introduced the notion of P-simple point as a verification method but also as a methodology to design parallel thinning algorithms.

More recently, one of the authors introduced in [5] a general framework for studying parallel homotopic thinning in spaces of any dimension. This framework, called critical kernels, is developped in the context of abstract simplicial or cubical complexes, but it also permits to prove properties of algorithms acting in $\mathbb{Z}^{D}$. In particular, the notion of crucial point is introduced in [7] and [6], for the 2D and the 3D case respectively, together with the proof that any set of non-crucial points can be removed in parallel from any object in $\mathbb{Z}^{D}$ without changing its topological characteristics.

In Sects. 3.4.1 to 3.4.4, we present a minimal set of notions needed to survey the critical kernels framework. Section 3.4.5 is devoted to parallel thinning in $\mathbb{Z}^{D}$, where results about critical kernels are used only to prove topological correctness. The reader who prefers to quickly implement algorithms may jump directly to this latter section.

\subsubsection{Cubical Complexes}

Intuitively, a cubical complex may be thought of as a set of elements having various dimensions (e.g. cubes, squares, edges, vertices) glued together according to certain rules (see Fig. 3.21d).

Let $\mathbb{Z}$ be the set of integers. We consider the families of sets $\mathbb{F}_{0}^{1}, \mathbb{F}_{1}^{1}$, such that $\mathbb{F}_{0}^{1}=\{\{a\} \mid a \in \mathbb{Z}\}, \mathbb{F}_{1}^{1}=\{\{a, a+1\} \mid a \in \mathbb{Z}\}$. A subset $f$ of $\mathbb{Z}^{D}, D \geqslant 2$, which is the Cartesian product of exactly $d$ elements of $\mathbb{F}_{1}^{1}$ and $(D-d)$ elements of $\mathbb{F}_{0}^{1}$ is called a face or a $d$-face in $\mathbb{Z}^{D}, d$ is the dimension of $f$, we write $\operatorname{dim}(f)=d$. See Fig. 3.21a,b for an illustration.

We denote by $\mathbb{F}^{D}$ the set composed of all faces in $\mathbb{Z}^{D}$. A $d$-face is called a point if $d=0$, a (unit) edge if $d=1$, a (unit) square if $d=2$, a (unit) cube if $d=3$. Observe that any non-empty intersection of faces is a face. For example, the intersection of two 2 -faces $A$ and $B$ may be either a 2 -face (if $A=B$ ), a 1-face, a 0-face, or the empty set.

Let $f$ be a face in $\mathbb{F}^{D}$. We set $\hat{f}=\left\{g \in \mathbb{F}^{D} \mid g \subseteq f\right\}$ and $\hat{f}^{*}=\hat{f} \backslash\{f\}$; we call $\hat{f}^{*}$ the boundary of $f$. Any $g \in \hat{f}$ is called a face of $f$. If $X$ is a finite set of faces in $\mathbb{F}^{D}$, we write $X^{-}=\bigcup\{\hat{f} \mid f \in X\}, X^{-}$is the closure of $X$. A finite set $X$ of faces in $\mathbb{F}^{D}$ is a complex (in $\mathbb{F}^{D}$ ) if $X=X^{-}$. If $Y \subseteq X$ and $Y$ is a complex, then we say that $Y$ is a subcomplex of $X$. In the sequel, the 
symbol $X$ will denote a complex in $\mathbb{F}^{D}$, and the symbol $f$ will denote a face of $X$.

See in Fig. 3.21d,e two examples of complexes, and in Fig. 3.21b,c examples of sets of faces that are not complexes. The complex in Fig. 3.21d is the closure of the complex in Fig. 3.21c.

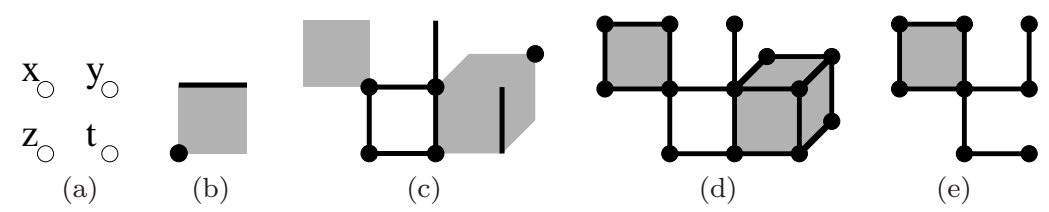

Fig. 3.21 (a) Four points in $\mathbb{Z}^{2}: x=(0,1) ; y=(1,1) ; z=(0,0) ; t=(1,0)$. (b) A graphical representation of the set of faces $\left\{f_{0}, f_{1}, f_{2}\right\}$, where $f_{0}=\{z\}=\{0\} \times\{0\}$ (a 0 -face), $f_{1}=\{x, y\}=\{0,1\} \times\{1\}$ (a 1-face), and $f_{2}=\{x, y, z, t\}=\{0,1\} \times\{0,1\}$ (a 2 -face). (b,c) A set of faces that is not a complex. (d,e) A set of faces that is a complex.

Let $d=\operatorname{dim}(f)$. We say that $f$ is a facet of $X$ or an $d$-facet of $X$ if there is no face $g \in X$ such that $f \in \hat{g}^{*}$, in other words, if $f$ is maximal for inclusion. We set $\operatorname{dim}(X)=\max \{\operatorname{dim}(f) \mid f \in X\}$. We say that $X$ is an $d$-complex if $\operatorname{dim}(X)=d$. We say that $X$ is pure if, for each facet $f$ of $X$, we have $\operatorname{dim}(f)=\operatorname{dim}(X)$. For example in Fig. 3.22, $X_{0}$ and $X_{2}$ are pure 2-complexes, whereas $X_{1}$ is a 2-complex which is not pure.

The operation of detachment allows us to remove a subset from a complex, while guaranteeing that the result is still a complex.

Let $Y \subseteq X$. We set $\operatorname{Detach}(Y, X)=(X \backslash Y)^{-}$. The set $\operatorname{Detach}(Y, X)$ is a complex which is the detachment of $Y$ from $X$. Figure 3.21e shows the detachment of $\hat{f}$ from $X$, where $X$ is the complex of Fig. 3.21d and $f$ is the 3 -face of $X$.

\subsubsection{Collapse and Simple Facets}

The collapse operation is an elementary topology-preserving transformation which has been introduced by J.H.C. Whitehead [39], and plays an important role in combinatorial topology. It can be seen as a discrete analogue of a continuous deformation (a strong deformation retract). Collapse is known to preserve the homotopy type.

Consider a pair $(f, g) \in X^{2}$. If $f$ is the only face of $X$ that strictly includes $g$, then $g$ is said to be free for $X$ and the pair $(f, g)$ is said to be a free pair for $X$. Note that, if $(f, g)$ is a free pair, then $f$ is necessarily a facet of $X$ and $\operatorname{dim}(g)=\operatorname{dim}(f)-1$.

Let $(f, g)$ be a free pair for $X$. Let $d=\operatorname{dim}(f)$. The complex $X \backslash\{f, g\}$ is an elementary collapse of $X$, or an elementary $d$-collapse of $X$. The pair 
$(f, g)$ is also called a free d-pair (for $X)$.

Let $Y$ be a complex. We say that $X$ collapses onto $Y$, and we write $X \searrow Y$, if $Y=X$ or if there exists a sequence of complexes $\left\langle X_{0}, \ldots, X_{\ell}\right\rangle$ such that $X_{0}=X, X_{\ell}=Y$, and $X_{i}$ is an elementary collapse of $X_{i-1}$, for each $i \in$ $\{1, \ldots, \ell\}$. See Fig. 3.22 for an illustration.
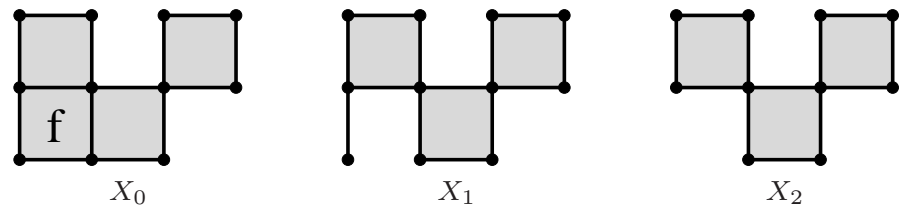

Fig. 3.22 $X_{0}$ : a pure 2-complex. $X_{1}$ : a complex such that $X_{0}$ collapses onto $X_{1}$; a free pair composed of a square and an edge has been removed. $X_{2}$ : a complex such that $X_{1}$ collapses onto $X_{2}$; (a free pair composed of an edge and a vertex has been removed), hence $X_{0}$ collapses onto $X_{2}$.

We give now a definition of a simple facet, it may be seen as a discrete analogue of the one given by T.Y. Kong in [28] which lies on continuous deformations in the $D$-dimensional Euclidean space.

Definition 6 ([5]). Let $f$ be a facet of $X$. We say that $\hat{f}$ and $f$ are simple for $X$ if $X$ collapses onto $\operatorname{Detach}(\hat{f}, X)$.

For example in Fig. 3.22, we have $X_{2}=\operatorname{Detach}\left(\hat{f}, X_{0}\right)$, and since $X_{0} \searrow$ $X_{2}$, the facet $f$ is simple for $X_{0}$.

The notion of attachment, as introduced by T.Y. Kong [27, 28], leads to a local characterization of simple facets. The attachment of $\hat{f}$ for $X$ is the $\operatorname{complex} \operatorname{Attach}(\hat{f}, X)=\hat{f}^{*} \cap[\operatorname{Detach}(\hat{f}, X)]$. In other words, a face $g$ is in $\operatorname{Attach}(\hat{f}, X)$ if $g$ is in $\hat{f}^{*}$ and if $g$ is a face of a facet $h$ distinct from $f$.

As an easy consequence of the above definitions, the facet $f$ is simple for $X$ if and only if $\hat{f}$ collapses onto $\operatorname{Attach}(\hat{f}, X)$. This property led us to introduce new characterizations of simple points in $2 \mathrm{D}, 3 \mathrm{D}$ and $4 \mathrm{D}$ [19].

\subsubsection{Critical Kernels}

Let us briefly recall the framework introduced by one of the authors (in [5]) for thinning, in parallel, discrete objects with the warranty that topology is preserved. We focus here on the two- and three-dimensional cases, but in fact the notions and results in this section are valid for complexes of arbitrary dimension. This framework is based solely on three notions: the notion of an essential face, which allows us to define the core of a face, and the notion of a critical face. 
Definition 7 ([5]). We say that $f$ is an essential face for $X$ if $f$ is precisely the intersection of all facets of $X$ that contain $f$. We denote by $E s s(X)$ the set composed of all essential faces of $X$. If $Y$ is a subcomplex of $X$ and $\operatorname{Ess}(Y) \subseteq \operatorname{Ess}(X)$, then we say that $Y$ is an essential subcomplex of $X$.

Observe that a facet of $X$ is necessarily an essential face for $X$. Observe also that, if $X$ and $Y$ are both pure $D$-complexes, then $Y$ is an essential subcomplex of $X$ whenever $Y$ is a subcomplex of $X$.

Definition 8 ([5]). Let $f \in \operatorname{Ess}(X)$. The core of $\hat{f}$ for $X$ is the complex $\operatorname{Core}(\hat{f}, X)=\cup\left\{\hat{g} \mid g \in \operatorname{Ess}(X) \cap \hat{f}^{*}\right\}$.

Definition 9 ([5]). Let $f \in X$. We say that $f$ and $\hat{f}$ are regular for $X$ if $f \in \operatorname{Ess}(X)$ and if $\hat{f}$ collapses onto $\operatorname{Core}(\hat{f}, X)$. We say that $f$ and $\hat{f}$ are critical for $X$ if $f \in \operatorname{Ess}(X)$ and if $f$ is not regular for $X$.

We set $\operatorname{Critic}(X)=\cup\{\hat{f} \mid f$ is critical for $X\}$, we say that $\operatorname{Critic}(X)$ is the critical kernel of $X$.

Figure 3.23 illustrates these definitions. In Fig. 3.23b, we see that $\hat{f}$ collapses onto the core of $f$, thus $f$ is regular; and that $\hat{g}$ does not collapse onto the core of $g$, thus $g$ is critical. Note that, in this complex, all facets (3-faces) are regular.

The following theorem is the most fundamental result concerning critical kernels. Note that the theorem holds whatever the dimension.

Theorem 3 ([5]). Let $Y$ be an essential subcomplex of $X$.

i) The complex $X$ collapses onto its critical kernel.

ii) If $Y$ contains the critical kernel of $X$, then $X$ collapses onto $Y$.

iii) If $Y$ contains the critical kernel of $X$, and if $Z$ is an essential subcomplex of $X$ such that $Y \subseteq Z$, then $Z$ collapses onto $Y$.

In Fig. 3.23, we show that the very notion of critical kernel can be seen as a powerful thinning algorithm, which consists of computing iteratively the critical kernel of the result of the preceding computation. Furthermore, Theorem 3ii tells us that any essential subcomplex $Y$ of $X$ that is "between" $X_{0}$ (Fig. 3.23a) and $X_{1}$ (Fig. 3.23d) is such that $X_{0}$ collapses onto $Y$. This is true, in particular, of any subcomplex $Y$ that is a pure 3-complex containing $X_{1}$. This property gives birth to a wide class of parallel thinning algorithms, where different criterions, based e.g. on geometrical notions, can be used in order to choose a particular set as the result of a single thinning step (see Sect. 3.4.5).

\subsubsection{Crucial Cliques and Faces}

In the image processing literature, a digital image is often considered as a set of pixels in 2D or voxels in 3D. A pixel (resp. a voxel) is an elementary square 


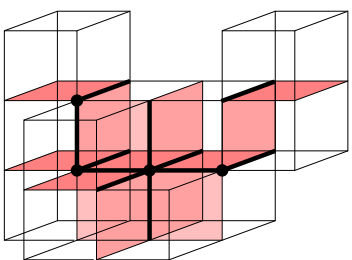

(a)

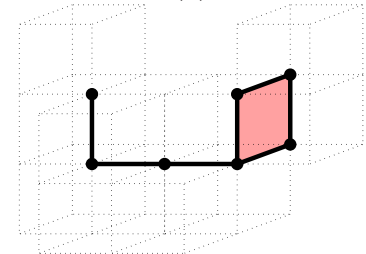

(d)

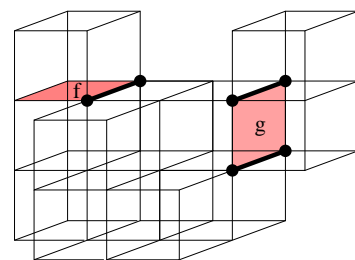

(b)

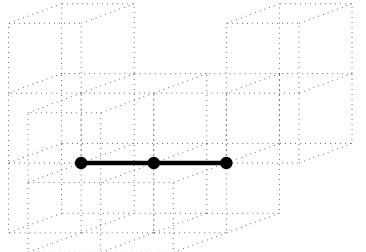

(e)

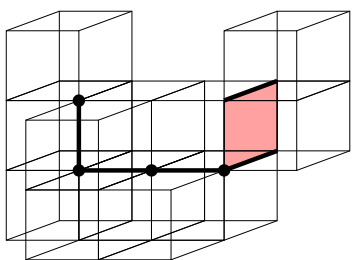

(c)

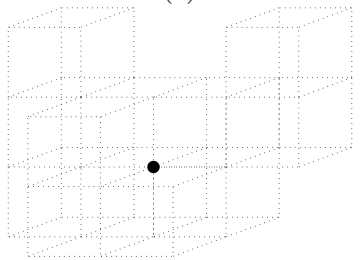

(f)

Fig. 3.23 (a): A 3-complex $X_{0}$, made of 12 cubes. The essential faces for $X_{0}$ that are not facets are highlighted. (b): Two essential 2-faces $f, g$ and their cores (in black). (c): $X_{0}$ and its critical faces (highlighted). (d): The critical kernel $X_{1}=\operatorname{Critic}\left(X_{0}\right)$. (e): $X_{2}=$ $\operatorname{Critic}\left(X_{1}\right)$. (f): $X_{3}=\operatorname{Critic}\left(X_{2}\right)=\operatorname{Critic}\left(X_{3}\right)$.

(resp. cube), thus an easy correspondence can be made between this classical view and the framework of cubical complexes. From now on, we consider only complexes whose facets are all $D$-faces, i.e., pure $D$-complexes.

Note that, if $X$ is a pure $D$-complex in $\mathbb{F}^{D}$ and if $f$ is a $D$-face of $X$, then $\operatorname{Detach}(\hat{f}, X)$ is a pure complex in $\mathbb{F}^{D}$. There is indeed an equivalence between the operation on complexes that consists of removing (by detachment) the closure of a simple $D$-face, and the removal of an 8-simple (resp. 26-simple) point in the framework of $2 \mathrm{D}$ (resp. 3D) digital topology (see [27, 28]).

When $X$ is a pure $D$-complex (e.g., a union of voxels in $\mathbb{F}^{3}$ ), the critical kernel of $X$ is not necessarily a pure $D$-complex (see Fig. 3.23d). The notion of crucial face, introduced in $[6,7]$, allows us to recover a pure $D$-subcomplex $Y$ of an arbitrary pure $D$-complex $X$, under the constraint that $X$ collapses onto $Y$.

Definition $10([6])$. A face $f$ in $X$ is a maximal critical face, or an $M$ critical face (for $X$ ), if $f$ is a facet of $\operatorname{Critic}(X)$.

The set of all the facets of $X$ that contain an M-critical face $f$ is called the crucial clique (for $X$ ) induced by $f$. Each facet in a crucial clique is called a crucial face.

Some 2D crucial cliques are illustrated in Fig. 3.24. The following corollary of Theorem 3 tell us that, informally speaking, a thinning step that preserves all non-simple pixels (voxels) and at least one pixel (voxel) in each crucial clique, preserves topology. 
(a)

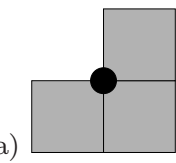

(b)

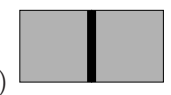

(c)

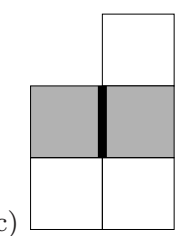

Fig. 3.24 Crucial cliques in $\mathbb{F}^{2}$ (represented in light gray): (a) induced by an M-critical 0 -face; (b,c) induced by an M-critical 1-face. The considered M-critical faces are in bold. The core of the M-critical face in (a,b) is empty, in (c) it consists of two 0-faces.

Corollary 2. Let $Y$ be a subcomplex of $X$ that is also a pure D-complex. If any critical $D$-face of $X$ and at least one $D$-face of each crucial clique of $X$ is in $Y$, then $X$ collapses onto $Y$.

During the process of thinning an object, we often want to keep certain faces like curve extremities for example, if we want to obtain a curvilinear skeleton. That is why we introduce the following definition in order to generalize the previous notions. Intuitively, the set $K$ corresponds to a set which is preserved by a thinning algorithm (a constraint set).

Definition 11 ([6]). Let $K$ be a set composed of facets of $X$. A subcomplex $C$ of $X$ is a crucial clique for $\langle X, K\rangle$ if $C$ is a crucial clique for $X$ such that $C \cap K=\emptyset$. In this case, each facet in $C$ is called a crucial face for $\langle X, K\rangle$.

\subsubsection{Parallel Thinning Algorithms}

In the sequel, we give a characterization of crucial points or pixels in $\mathbb{Z}^{2}$, which can be checked in a quite simple manner with the help of masks. Thanks to this characterization, one can easily implement parallel thinning algorithms that are guaranteed to preserve topology. The interested reader is referred to $[5,7,6,8]$ for the proofs of the stated properties ${ }^{2}$. Implementations (in source code) are available on the critical kernels web site ${ }^{3}$. We emphasize that no representation of cubical complexes is used for computing this characterization and thinning methods based on it: both inputs and outputs, as well as intermediate results, are mere binary images (i.e., subsets of $\mathbb{Z}^{D}$ ). For the sake of simplicity, we limit ourselves to the $2 \mathrm{D}$ case, the reader can find a similar characterization for the $3 \mathrm{D}$ case in $[6]$.

${ }^{2}$ Note that the characterization that we use in this chapter for the $2 \mathrm{D}$ case is actually derived from the ones of [6], which deals with $3 \mathrm{D}$. This allows us to present a characterization that is simpler than the one proposed in [7].

3 http://www.esiee.fr/ info/ck 


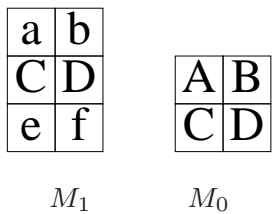

Fig. 3.25 Masks for 1-crucial $\left(M_{1}\right)$ and 0 -crucial $\left(M_{0}\right)$ points.

The masks $M_{1}, M_{0}$ are given in Fig. 3.25. For the mask $M_{1}$, we also consider the mask obtained from it by applying a $\pi / 2$ rotation: we get 3 masks ( 2 for $M_{1}$, and 1 for $M_{0}$ ).

Definition 12. Let $X \subseteq \mathbb{Z}^{2}$, and let $M$ be a set of points of $X$.

1) The set $M$ matches the mask $M_{1}$ if:

i) $M=\{C, D\}$; and

ii) the points $C, D$ are simple for $X$; and

iii) the sets $\{a, b\} \cap X$ and $\{e, f\} \cap X$ are either both empty or both nonempty.

2) The set $M$ matches the mask $M_{0}$ if:

i) $M=\{A, B, C, D\} \cap X$; and

ii) the points in $M$ are simple and not matched by $M_{1}$; and

iii) at least one of the sets $\{A, D\},\{B, C\}$ is a subset of $M$.

In the following, the set $K$ plays the role of a constraint set (see Sect. 3.2.4). There exists a "natural" one-to-one correspondence between the subsets of $\mathbb{Z}^{D}$ and the pure $D$-complexes in $\mathbb{F}^{D}$ (see $[6,7]$ ). Namely, with each point (pixel, voxel) of $\mathbb{Z}^{D}$ we associate a facet of $\mathbb{F}^{D}$ (unit square, unit cube). We extend our vocabulary accordingly: for instance, we say that a point $x \in X$ is crucial whenever the corresponding facet in the corresponding complex is crucial.

Property 1. Let $X \subseteq \mathbb{Z}^{2}, K \subseteq X$, and let $M$ be a set of points in $X \backslash K$ that are 8-simple for $X$.

Then, $M$ is a crucial clique for $\langle X, K\rangle$ if and only if $M$ matches the mask $M_{0}$ or the mask $M_{1}$.

An illustration is given in Fig. 3.26. From Corollary 2, we deduce that a parallel thinning step that preserves all critical (i.e., non-simple) points and at least one point of each crucial clique, preserves topology.

The simplest parallel thinning algorithm based on crucial points is the following one. It consists of iteratively detecting the points that are simple and not crucial (with respect to the current object and a possibly empty constraint set), and removing them in parallel. This algorithm makes no arbitrary choice: whenever a crucial clique is detected, all its points are preserved. 

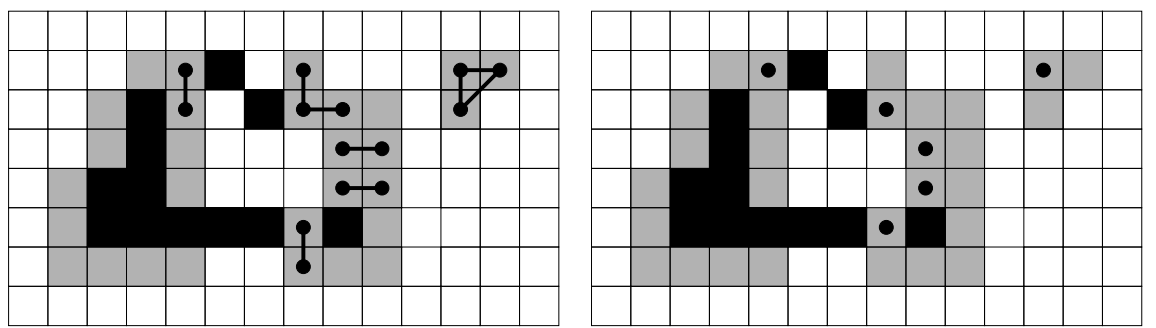

Fig. 3.26 Illustration of crucial points (pixels). Left: the simple points are in gray, the crucial points are marked by a black disk. The couples of black disks that are linked by a bar, in the biggest connected component, represent all the crucial cliques that are dectected by the mask $M_{1}$. The triplet of black disks that are linked by a triangle, in the smallest connected component, represents a crucial clique that is dectected by mask $M_{0}$. All simple points that are not crucial may be removed in parallel by a topology-preserving algorithm. Right: the crucial points marked by a black disk constitute a set of points that is sufficient to ensure topology preservation. All other simple points may be safely removed in parallel.

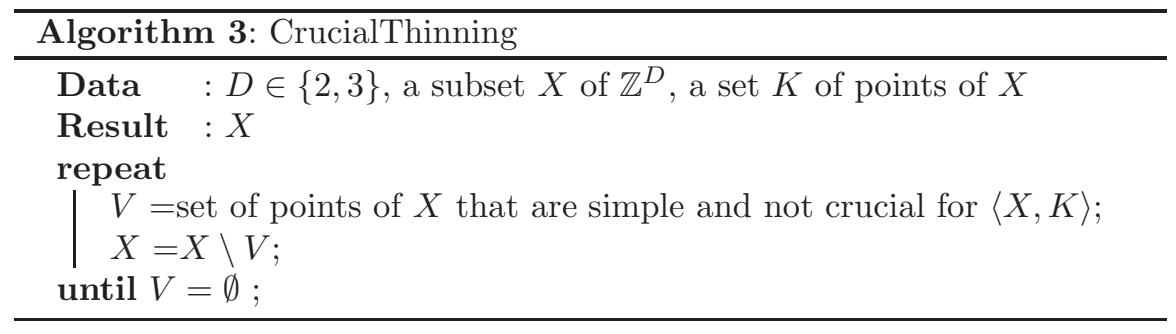

In $[7,6]$, we provide various algorithms based on the same principle, that compute different kinds of skeletons: curvilinear of surface skeletons in 3D, skeletons that are guaranteed to contain the medial axis, minimal skeletons, asymetric skeletons, skeletons of three-dimensional objects made of surfels...

Back to guided thinning, we show with the next algorithm how to use the notion of crucial point in order to avoid arbitrary choices when several candidate points share the same priority. The result of the following procedure is thus uniquely defined, given any shape and any priority function.

By construction, at each iteration of Algorithm 4, the current set $X$ has the same topology as the initial object. By "stacking" these sets that are nested in each other, we can build a function that is a compact representation of this family of thinnings. The simplest way to do this consists of defining a function $F$ that associates with each point $x$ of $X$, the number of the iteration where $x$ is deleted, or $+\infty$ whenever $x$ is still in the final set. Hence, thresholding $F$ at any integer level provides one of the thinnings. Instead of the number of the iteration, we can indeed choose any number that increases at each iteration. This is not necessarily the case of the number $\pi$, but a slight modification of the algorithm allows us to compute a function which is closely related to the priority function used as input. This leads us to Algorithm 5. 

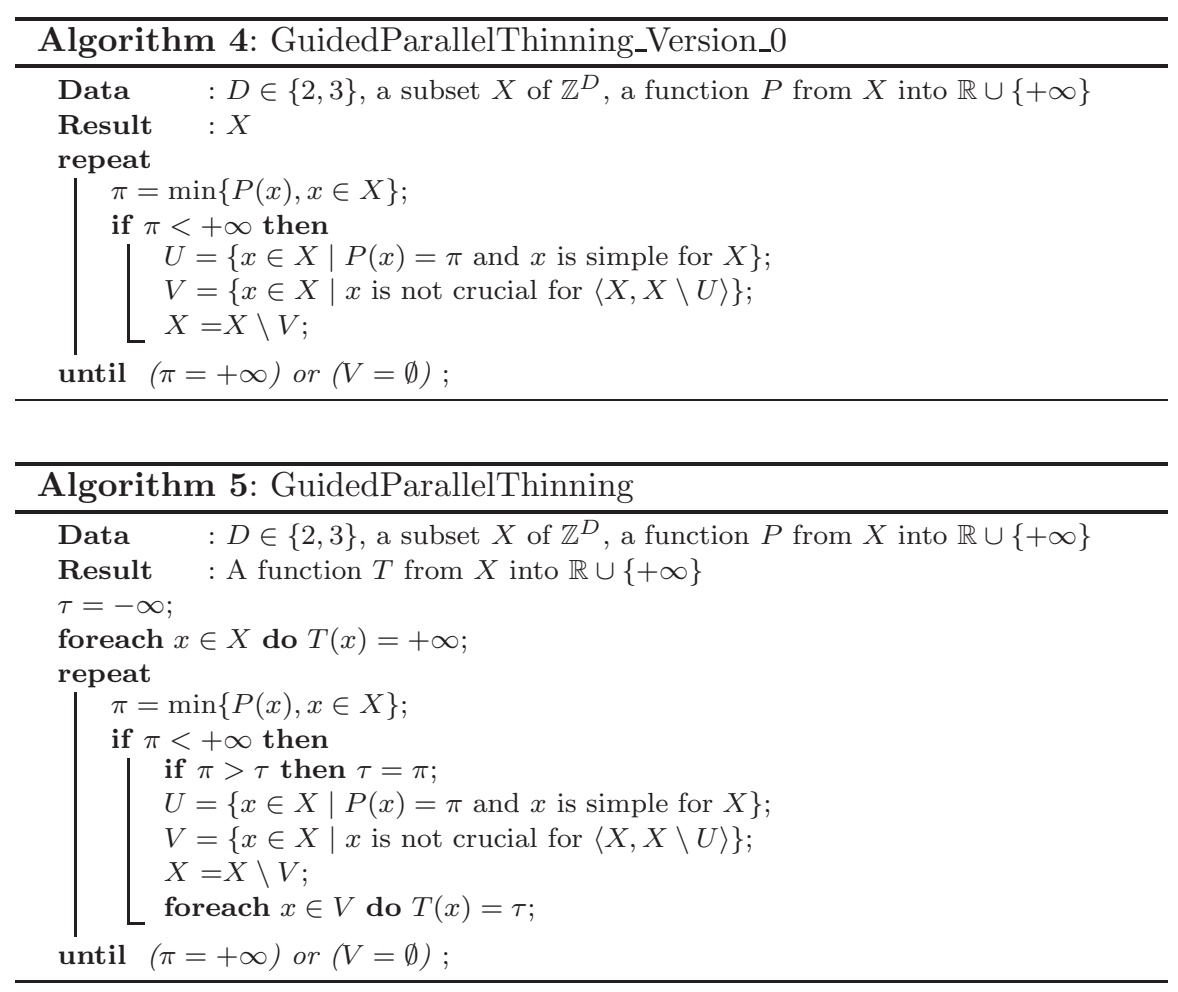

Figure 3.27 shows, on the right, an example of function computed by Algorithm GuidedParallelThinning, using the same input shape $X$ as in Fig. 3.8, and the priority function $P R_{X}$ defined in Sect. 3.2.6 (depicted on the left).

As for Algorithm GuidedThinning, it is possible to implement this algorithm in $O(n \log n)$ or $O(n)$ time complexity, depending on the nature of the priority function.
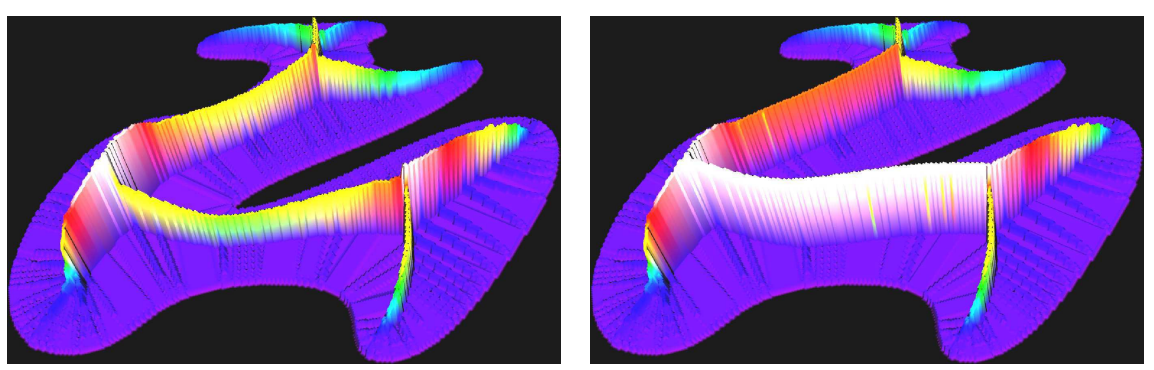

Fig. 3.27 Left: a visualization of the map $P R_{X}$, for the same shape $X$ as in Fig. 3.8. Right: the result of Algorithm GuidedParallelThinning. 


\subsection{Perspectives}

All the algorithms presented in this chapter work on images defined on $\mathbb{Z}^{D}$. They fit in the framework, called digital topology, pionneered by A. Rosenfeld [34]. The success of digital topology is mainly due to its simplicity, especially for the $2 \mathrm{D}$ case. However, topological properties in higher dimensions are not easily handled in this framework.

Besides, in Sects. 3.4.1-3.4.4, we described a framework based on cubical complexes in which topological notions are defined quite naturally. Abstract (cubical) complexes have been promoted in particular by V. Kovalevsky [30], in order to provide a sound topological basis for image analysis. The cubical complexes framework allows for retrieving the results obtained using digital topology, providing a better understanding of these results. Furthermore, new properties can be proved and new methods can be developped in this framework, as showed by the example of critical kernels for the study of parallel homotopic thinning in any dimension.

Further developments are needed to fully explore the possibilities and the benefits of working directly on objects which are general cubical complexes, and not only pure ones as it is the case in this chapter. In applications, this should lead in particular to easier characterization, detection and analysis of lower-dimensional structures, such as curves in $2 \mathrm{D}$ and $3 \mathrm{D}$, and surfaces in $3 \mathrm{D}$.

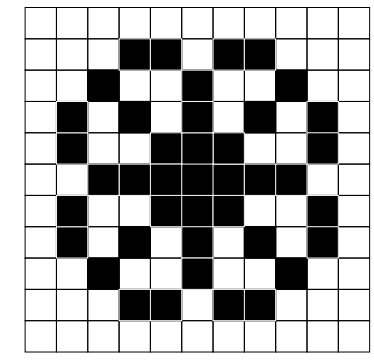

Fig. 3.28 An ultimate skeleton (all pixels are non-simple) that is not thin.

To illustrate this, let us consider the example of Fig. 3.28. In the continuous framework, the skeleton of a bounded $D$-dimensional object always has a dimension that is at most $D-1$. That is, the skeleton of any object in $2 \mathrm{D}$ is made of curves (1D) and points (0D). Figure 3.28 is a classical example showing that this property of thinness is not always true in the digital topology framework.

However it is indeed possible to provide thinness guarantees in the cubical complex framework. Consider the following thining scheme, based on the collapse operation (see Sect. 3.4.2). Each thinning step is decomposed into 


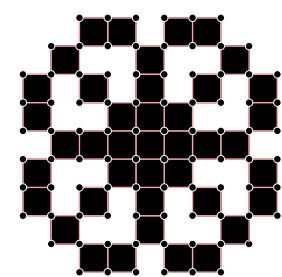

(a)

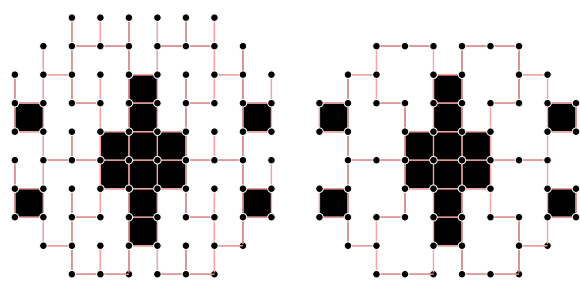

(b) (c)

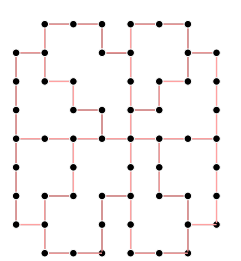

(d)

Fig. 3.29 Illustration of a thinning scheme based on collapse (see text).

four (in 2D) substeps corresponding to the four principal directions of the grid, named north $(\mathrm{N})$, south $(\mathrm{S})$, west $(\mathrm{W})$, and east $(\mathrm{E})$. A north free $k$ pair is a pair of faces $(f, g)$ such that $f$ is the only face that strictly includes $g$, $\operatorname{dim}(f)=k$, and $g$ is on the north of $f$. In the substep $\mathrm{N}$, only north free pairs are considered. All north free 2-pairs are marked, and then removed in parallel (see Fig. 3.29a,b). Then, all north free 1-pairs are marked, and then removed in parallel (see Fig. 3.29b,c). The thinning scheme iterates such steps until no more free pair can be found in a complete step (NSWE). The topological soundness of this scheme can easily be proved. In Fig. 3.29d, we show the final result obtained from the object of Fig. 3.29a. Observe that the obtained skeleton is only composed of 0 -faces and 1-faces, and can indeed be interpreted as a set of curves. This thinness property may also be proved in the general case. Of course, additional conditions may be added to this scheme in order to preserve geometrical features such as curve extremities (see [15]).

The cross-section topology approach presented in Sect. 3.3 can also be adapted to the case of functions defined on cubical complexes, and benefit from the ease of defining sound parallel topological operators, based on the critical kernels main property (Theorem 3), or directly on the collapse operation.

\subsection{Conclusion}

We have seen that it is possible to design topological operators acting on binary 2D and 3D images and also on grayscale images, which are well defined, have proven topological properties and can be implemented through efficient algorithms.

We studied operators that transform an image while preserving its topological characteristics, and also operators that selectively modify these characteristics in order to achieve some filtering, segmentation or restoration. 
Thanks to the general scheme of guided thinning that we promote in this chapter, the geometrical features of the processed objects may be taken into account in a flexible way, through the choice of adapted priority functions (e.g. distance maps) and constraint sets.

In addition to the sequential approach, which has the advantage of being simple but the drawback of needing arbitrary decisions to be made, we present a tractable way to design sound parallel homotopic thinning algorithms, based on the critical kernels framework. We show that the guided thinning strategy, in particular, may benefit from this approach and result in a well-defined and flexible thinning scheme.

The critical kernels framework is based on cubical complexes, that we shortly presented in Sects. 3.4.1-3.4.4. In this chapter, cubical complexes were used only to prove topological properties of algorithms acting in $\mathbb{Z}^{D}$. Further developments are needed to build a coherent set of image processing tools based on cubical complexes, dealing with both binary and grayscale images, encompassing and extending the set of digital topology tools.

\section{Acknowledgments}

This work has been partially supported by the "ANR BLAN07-2_184378 MicroFiss" project and the "ANR-2010-BLAN-0205 Kidico" project.

\section{References}

1. Aktouf, Z., Bertrand, G., Perroton, L.: A three-dimensional holes closing algorithm. Pattern Recognition Letters 23(5), 523-531 (2002)

2. Attali, D., Boissonnat, J., Edelsbrunner, H.: Stability and computation of the medial axis - a state-of-the-art report. In: Mathematical Foundations of Scientific Visualization, Computer Graphics, and Massive Data Exploration, pp. 109-125. Springer-Verlag (2009)

3. Bertrand, G.: Simple points, topological numbers and geodesic neighborhoods in cubic grids. Pattern Recognition Letters 15, 1003-1011 (1994)

4. Bertrand, G.: On P-simple points. Comptes Rendus de l'Académie des Sciences, Série Math. I(321), 1077-1084 (1995)

5. Bertrand, G.: On critical kernels. Comptes Rendus de l'Académie des Sciences, Série Math. I(345), 363-367 (2007)

6. Bertrand, G., Couprie, M.: New 3D parallel thinning algorithms based on critical kernels. In: Discrete geometry for computer imagery. Lecture Notes in Computer Science, vol. 4245 , pp. 580-591. Springer (2006)

7. Bertrand, G., Couprie, M.: Two-dimensional thinning algorithms based on critical kernels. J. of Mathematical Imaging and Vision 31(1), 35-56 (2008)

8. Bertrand, G., Couprie, M.: On parallel thinning algorithms: minimal non-simple sets, P-simple points and critical kernels. J. of Mathematical Imaging and Vision 35(1), 23-35 (2009) 
9. Bertrand, G., Everat, J., Couprie, M.: Image segmentation through operators based upon topology. J. of Electronic Imaging 6(4), 395-405 (1997)

10. Bertrand, G., Malandain, G.: A new characterization of three-dimensional simple points. Pattern Recognition Letters 15(2), 169-175 (February 1994)

11. Blum, H.: An associative machine for dealing with the visual field and some of its biological implications. Biol. prototypes and synthetic systems 1, 244-260 (1961)

12. Blum, H.: A transformation for extracting new descriptors of shape. In: Models for the Perception of Speech and Visual Form, pp. 362-380. MIT Press (1967)

13. Borgefors, G.: Distance transformations in digital images. Computer Vision, Graphics and Image Processing 34, 344-371 (1986)

14. Chaussard, J., Couprie, M., Talbot, H.: Robust skeletonization using the discrete lambda-medial axis. Pattern Recognition Letters pp. 1-10 (2011), to appear

15. Chaussard, J.: Topological tools for discrete shape analysis. Ph.D. thesis, Université Paris-Est (December 2010)

16. Chazal, F., Lieutier, A.: The $\lambda$-medial axis. Graphical Models 67(4), 304-331 (2005)

17. Couprie, M.: Note on fifteen 2D parallel thinning algorithms. Tech. Rep. IGM2006-01, Université de Marne-la-Vallée (2006)

18. Couprie, M., Bertrand, G.: Topology preserving alternating sequential filter for smoothing 2D and 3D objects. J. of Electronic Imaging 13(4), 720-730 (2004)

19. Couprie, M., Bertrand, G.: New characterizations of simple points in 2D, 3D and 4D discrete spaces. IEEE Trans. on Pattern Analysis and Machine Intelligence 31(4), 637-648 (2009)

20. Couprie, M., Bezerra, F., Bertrand, G.: Topological operators for grayscale image processing. J. of Electronic Imaging 10(4), 1003-1015 (2001)

21. Daragon, X., Couprie, M.: Segmentation topologique du neo-cortex cérébral depuis des données IRM. In: procs. congrès RFIA. vol. 3, pp. 809-818 (2002)

22. Dokládal, P., Bloch, I., Couprie, M., Ruijters, D., Urtasun, R., Garnero, L.: Segmentation of 3D head MR images using morphological reconstruction under constraints and automatic selection of markers. Pattern Recognition 36, 2463-2478 (2003)

23. Fabbri, R., Costa, L.D.F., Torelli, J., Bruno, O.: 2D Euclidean distance transform algorithms: a comparative study. ACM Computing Surveys 40(1), 1-44 (2008)

24. Fourey, S., Malgouyres, R.: A concise characterization of 3D simple points. Discrete Applied Mathematics 125(1), 59-80 (2003)

25. Hall, R.: Tests for connectivity preservation for parallel reduction operators. Topology and its Applications 46(3), 199-217 (1992)

26. Kong, T.: On the problem of determining whether a parallel reduction operator for $\mathrm{n}$ dimensional binary images always preserves topology. In: procs. SPIE Vision Geometry II. vol. 2060, pp. 69-77 (1993)

27. Kong, T.: On topology preservation in $2 \mathrm{D}$ and $3 \mathrm{D}$ thinning. Int. J. on Pattern Recognition and Artificial Intelligence 9, 813-844 (1995)

28. Kong, T.: Topology-preserving deletion of 1's from 2-, 3- and 4-dimensional binary images. In: procs. DGCI, LNCS. vol. 1347, pp. 3-18 (1997)

29. Kong, T., Rosenfeld, A.: Digital topology: introduction and survey. Computer Vision, Graphics and Image Processing 48, 357-393 (1989)

30. Kovalevsky, V.: Finite topology as applied to image analysis. Computer Vision, Graphics and Image Processing 46, 141-161 (1989)

31. Ma, C.: On topology preservation in 3D thinning. Computer Vision, Graphics and Image Processing 59(3), 328-339 (1994)

32. Malandain, G., Bertrand, G., Ayache, N.: Topological segmentation of discrete surfaces. Int. J. of Computer Vision 10 2, 183-197 (1993)

33. Ronse, C.: Minimal test patterns for connectivity preservation in parallel thinning algorithms for binary digital images. Discrete Applied Mathematics 21(1), 67-79 (1988)

34. Rosenfeld, A.: Digital topology. American Mathematical Monthly 86, 621-630 (1979)

35. Rosenfeld, A., Pfaltz, J.: Distance functions on digital pictures. Pattern Recognition 1, 33-61 (1968) 
36. Rutovitz, D.: Pattern recognition. Journal of the Royal Statistical Society 129, 504-530 (1966)

37. Stefanelli, S., Rosenfeld, A.: Some parallel thinning algorithms for digital pictures. J. of the Association for Computer Machinery 18(2), 255-264 (April 1971)

38. Talbot, H., Vincent, L.: Euclidean skeletons and conditional bisectors. In: Procs. VCIP'92, SPIE. vol. 1818, pp. 862-876 (1992)

39. Whitehead, J.: Simplicial spaces, nuclei and $m$-groups. Proceedings of the London Mathematical Society 45(2), 243-327 (1939) 


\section{Subject Index}

$\kappa$-destructible point, 21

$\lambda$-medial axis, 9

$n$-connected components, 2

$n$-connected points, 2

$n$-path, 2

attachment, 27

border points, 5

collapse operation, 26

complex, 25

connected components, 3, 6

connectivity numbers, $4,14,18$

critical kernel, 28

cross-section at level $k, 16$

CrucialThinning Algorithm, 32

detachment, 26

direct neighborhood, 10

distance map, 7, 16

elementary homotopic thinning, 17

essential face, 27

essential subcomplex, 28 extended projection, 10

GuidedParallelThinning Algorithm, 33

GuidedThinning Algorithm, 8

HoleClosing Algorithm, 16

homotopic thinning, 7, 18

interior point, 5

isolated point, 5

isthmus, 5,6

matching the mask, 31

maximal critical face, 29

neighborhood relations, 2

separating point, 22

simple point, $3-5$

subcomplex, 25

tunnel (hole), 3, 6, 14

ultimate homotopic thinning, 20 